\title{
Simple Intrinsic Simulation of Cellular Automata in Oritatami Molecular Folding Model
}

\author{
Daria Pchelina ${ }^{1}$, Nicolas Schabanel ${ }^{2 \star}$, Shinnosuke Seki ${ }^{3 \star \star}$, and Yuki Ubukata ${ }^{4}$ \\ 1 École Normale Supérieure de Paris, France \\ 2 École Normale Supérieure de Lyon (LIP UMR5668, MC2, ENS de Lyon), France \\ 3 U. of Electro-Communications, 1-5-1 Chofugaoka, Chofu, Tokyo, 1828585, Japan. \\ ${ }^{4}$ NTT DATA Corporation, Tokyo, Japan
}

\begin{abstract}
The Oritatami model was introduced by Geary et al (2016) to study the computational potential of RNA cotranscriptional folding as first shown in wet-lab experiments by Geary et al (Science 2014). In Oritatami model, a molecule grows component by component (named beads) into the triangular grid and folds as it grows. More precisely, the $\delta$ last nascent beads are free to move and adopt the positions that maximizes the number of bonds with the current folded structure. Geary et al (2018) proved that the Oritatami model is capable of efficient Turing universal computation using a complicated construction that simulates Turing machines via tag systems. We propose here a simple Oritatami system which intrinsically simulates arbitrary $1 \mathrm{D}$ cellular automata. Being intrinsic, our simulation emulates the behavior of cellular automata in a readable way and in time linear in space and time of the simulated automaton. Oritatami model has proven to be a fruitful framework to study molecular reconfigurability. Our construction relies on the development of new mecanisms which are simple enough that we believe that some simplification of them may be implemented in the wet lab. An implementation of our construction can be downloaded for testing.
\end{abstract}

Keywords: Molecular Self-assembly · Co-transcriptional folding · Intrinsic universality · Cellular automata · Turing universality

\section{Introduction}

DNA computing encompasses the field which tries to implement computation at the molecular levels. A recent example is [16] which implements arbitrary 6-bits cellular automata onto DNA nanotubes, realising a first DNA-based universal computer. This field success was built by going back and forth between theory, models and experiments. The Oritatami model was introduced in 2016 by [4]

* His work is supported in part by the CNRS grants MOPREXPROGMOL and AMARP from the Mission pour l'interdisciplinarité

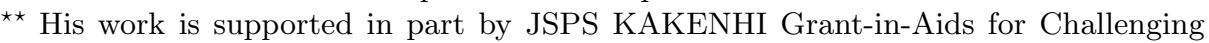
Research (Exploratory) No. 18K19779 and JST Program to Disseminate Tenure Tracking System, MEXT, Japan, No. 6F36. 
to study the computational potential of RNA cotranscriptional folding as first shown in wet-lab experiments by [5].

In Oritatami systems, we consider a finite set of bead types, and a periodic sequence of beads, each of a specific bead type. Beads are attracted to each other according to a fixed symmetric relation, and in any folding (configuration), whenever two beads attracted to each other are found at adjacent positions, a bond is formed between them. At each step, the latest few beads in the sequence are allowed to explore all possible positions, and we keep only those positions that minimise the energy, or otherwise put, those positions that maximise the number of bonds in the folding. "Beads" are a metaphor for domains, i.e. subsequences, in RNA and DNA. Oritatami model has proven to be a fruitful framework to study molecular reconfigurability, one of the most promising direction to reduce error in wetlab molecular implementation as error might be erased by reconfiguration later on. Indeed, programming Oritatami systems consists in designing molecules whose shape changes depending on its context, hence achieving some form of reconfiguration. Other models studying molecular reconfiguration include nubots [15] and signal passing tile assembly $[10,11]$. Previous work on Oritatami includes among others the implementation of a binary counter [4], the Heighway dragon fractal [7], folding of shapes at small scale [2], NP-hardness of the rule minimization $[6,9]$, a study of its parameters [12], and polynomial-time Turing machine simulation [3].

Our contribution. Geary et al's universality result in [3] relies on a complicated construction that simulates Turing machines via tag systems $[1,17]$. We propose here a simple Oritatami system which intrinsically simulates arbitrary 1D cellular automata. Being intrinsic $[8,14]$, our simulation emulates the behavior of cellular automata in a readable way and in time which is linear in the space and time of the simulated automaton. Precisely,

Theorem 1 (Main result). There is a universal finite set of 183 bead types $\mathcal{B}$ such that for any $1 D$ cellular automaton $A$ with $Q$ states and radius $r$, there is a delay-2 Oritatami system with bead types in $\mathcal{B}$ and periodic transcript with period precisely

$$
\frac{71}{3}\left((3+q) \cdot 2\left(Q_{r}\right)^{2}+8(2 q \bmod 3)\right)+10 q+610 \sim \frac{142}{3}\left(Q_{r}\right)^{2} \log _{2} Q_{r}
$$

that simulates $A$ intrinsically with the supercell shaped as a lozenge with sides of size $O\left(\left(Q_{r}\right)^{2} \log Q_{r}\right)$, where $q=\left\lceil\log _{2}\left(2 Q^{2 r+1}\right)\right\rceil$ and $Q_{r}=2^{q} \leqslant 4 Q^{2 r+1}$.

This improves the previous construction in [3] as the number of bead types is only 183 (instead of 542) and the delay is 2 (instead of 3). Furthermore, our construction relies on the development of new mechanisms which are simple enough to believe that some simplification of them may be implemented in the wet lab.

An implementation of our construction can be downloaded for testing [13]. 


\section{Model and Preliminary results}

\subsection{Oritatami model}

Let $B$ be a finite set of bead types. A configuration $c$ of a bead type sequence $p \in B^{*} \cup B^{\mathbb{N}}$ is a directed self-avoiding path $c_{0} c_{1} c_{2} \cdots$ in the triangular lattice $\mathbb{T},{ }^{5}$ where for all integer $i$, the vertex $c_{i}$ of $c$ is labeled by $p_{i}$ and refers to the position in $\mathbb{T}$ of the $(i+1)$-th bead in the configuration. A partial configuration of $p$ is a configuration of a prefix of $p$. The class of all the configurations obtained by applying an isometry of $\mathbb{T}$ to a given configuration is called a conformation.

For any partial configuration $c$ of some sequence $p$, an elongation of $c$ by $k$ beads (or $k$-elongation) is a partial configuration of $p$ of length $|c|+k$ extending by $k$ positions the self-avoiding path of $c$. We denote by $\mathcal{C}_{p}$ the set of all partial configurations of $p$ (the index $p$ will be omitted whenever it is clear from the context). We denote by $c^{\triangleright k}$ the set of all $k$-elongations of a partial configuration $c$ of sequence $p$.

Oritatami systems. An oritatami system $\mathcal{O}=(p, \phi, \delta)$ is composed of $(1)$ a (possibly infinite) bead type sequence $p$, called the transcript, (2) an attraction rule, which is a symmetric relation $\square \subseteq B^{2}$, and (3) a parameter $\delta$ called the delay. $\mathcal{O}$ is said periodic if $p$ is infinite and periodic. Periodicity ensures that the "program" $p$ embedded in the oritatami system is finite (does not hardcode any specific behavior) and at the same time allows arbitrary long computation.

We say that two bead types $a$ and $b$ attract each other when $a b b$. Furthermore, given a (partial) configuration $c$ of a bead type sequence $q$, we say that there is a bond between two adjacent positions $c_{i}$ and $c_{j}$ of $c$ in $\mathbb{T}$ if $q_{i} \$ q_{j}$ and $|i-j|>1$. The number of bonds of configuration $c$ of $q$ is denoted by $H(c)=\mid\left\{(i, j): c_{i} \sim c_{j}, j>i+1\right.$, and $\left.q_{i} q_{j}\right\} \mid$.

Oritatami dynamics. The folding of an oritatami system is controlled by the delay $\delta$. Informally, the configuration grows from a seed configuration (the input), one bead at a time. This new bead adopts the position(s) that maximize(s) the potential number of bonds the configuration can make when elongated by $\delta$ beads in total. This dynamics is oblivious as it keeps no memory of the previously preferred positions [3].

Formally, given an oritatami system $\mathcal{O}=(p, \phi, \delta)$ and a seed configuration $\sigma$ of a seed bead type sequence $s$, we denote by $\mathcal{C}_{\sigma, p}$ the set of all partial configurations of the sequence $s \cdot p$ elongating the seed configuration $\sigma$. The considered dynamics $\mathscr{D}: 2^{\mathcal{C}_{\sigma, p}} \rightarrow 2^{\mathcal{C}_{\sigma, p}}$ maps every subset $S$ of partial configurations of length $\ell$ elongating $\sigma$ of the sequence $s \cdot p$ to the subset $\mathscr{D}(S)$ of partial

\footnotetext{
${ }^{5}$ The triangular lattice is defined as $\mathbb{T}=\left(\mathbb{Z}^{2}, \sim\right)$, where $(x, y) \sim(u, v)$ if and only if $(u, v) \in \cup_{\epsilon= \pm 1}\{(x+\epsilon, y),(x, y+\epsilon),(x+\epsilon, y+\epsilon)\}$. Every position $(x, y)$ in $\mathbb{T}$ is mapped in the euclidean plane to $x \cdot \overrightarrow{\mathrm{e}}+y \cdot \overrightarrow{\mathrm{sw}}$ using the vector basis $\overrightarrow{\mathrm{e}}=(1,0)$ and $\overrightarrow{\mathrm{sW}}=$ RotateClockwise $\left(\overrightarrow{\mathrm{e}}, 120^{\circ}\right)=\left(-\frac{1}{2},-\frac{\sqrt{3}}{2}\right)$.
} 
configurations of length $\ell+1$ of $s \cdot p$ as follows:

$$
\mathscr{D}(S)=\bigcup_{c \in S} \arg \max \left(\max _{\gamma \in c^{\triangleright 1}} H(\eta)\right)
$$

The possible configurations at time $t$ of the oritatami system $\mathcal{O}$ are the elongations of the seed configuration $\sigma$ by $t$ beads in the set $\mathscr{D}^{t}(\{\sigma\})$.

We say that the oritatami system is deterministic if at all time $t, \mathscr{D}^{t}(\{\sigma\})$ is either a singleton or the empty set. In this case, we denote by $c^{t}$ the configuration at time $t$, such that: $c^{0}=\sigma$ and $\mathscr{D}^{t}(\{\sigma\})=\left\{c^{t}\right\}$ for all $t>0$; we say that the partial configuration $c^{t}$ folds (co-transcriptionally) into the partial configuration $c^{t+1}$ deterministically. In this case, at time $t$, the $(t+1)$-th bead of $p$ is placed in $c^{t+1}$ at the position that maximises the number of bonds that can be made in a $\delta$-elongation of $c^{t}$.

\subsection{Sweeping 2-fan-in 2-fan-out cellular automata}

Our construction simulates intrinsically the space-time diagrams of a specific type of one-way cellular automata where each cell has fan-in and fan-out 2 as shown on Fig. 8a, very similar to the gates implemented in [16]. Formally, a 2-fan-in 2-fan-out automaton (2FA) $\mathcal{A}$ is given by its set of states $[Q]=$ $\{0, \ldots, Q-1\}$ and its transition function $f:[Q]^{2} \rightarrow[Q]^{2}$. A finite configuration of $\mathcal{A}$ is an even-length word $c \in[Q]^{*}$, and its image by $\mathcal{A}$ is $c^{\prime}=F(c)$ where $\left(c_{2 i}^{\prime}, c_{2 i+1}^{\prime}\right)=f\left(c_{2 i-1}, c_{2 i}\right)$ for $i=0 . . \frac{|c|}{2}-1$, with the convention that $c_{-1}=$ $c_{|c|}=0$. Classically, any 1D cellular automaton with $Q$ states and radius $r$ can be simulated intrinsically by a $2 \mathrm{FA}$ with $Q^{2 r+1}$ states using a time rescaling by $r$ (no space rescaling is needed) as shown on Fig. 8a.

Our construction simulates intrinsically any 2FA by sweeping down (even time step) and up (odd time step), see Fig. 8b. As a consequence, every other step, the two inputs are read in reverse order and the transition function is applied with its arguments exchanged. Formally a configuration $(c, d)$ of a sweeping 2FA (S2FA) ([Q],f) consists in an even-length word $c \in[Q]^{*}$ together with a direction $d \in\{\uparrow, \downarrow\}$, and has the following dynamics: $F(c, \downarrow)=\left(c^{\prime}, \uparrow\right)$ where $\left(c_{2 i}^{\prime}, c_{2 i+1}^{\prime}\right)=f\left(c_{2 i-1}, c_{2 i}\right)$ for $i=0 . . \frac{|c|}{2}-1 ; F(c, \uparrow)=\left(c^{\prime}, \downarrow\right)$ where $\left(c_{2 i+1}^{\prime}, c_{2 i}^{\prime}\right)=f\left(c_{2 i}, c_{2 i-1}\right)$ for $i=0 . .|c| / 2-1$. Clearly, any 2FA $([Q], f)$ can be simulated intrinsically in real time by the S2FA $([Q] \times\{\uparrow, \downarrow\}, g)$ where $g((x, \uparrow),(y, \uparrow))=\left(\left(x^{\prime}, \downarrow\right),\left(y^{\prime}, \downarrow\right)\right)$ where $\left(x^{\prime}, y^{\prime}\right)=f(x, y)$; and $g((x, \downarrow),(y, \downarrow))=$ $\left(\left(x^{\prime}, \uparrow\right),\left(y^{\prime}, \uparrow\right)\right)$ where $\left(y^{\prime}, x^{\prime}\right)=f(y, x)$.

From now on, we consider a S2FA $\mathcal{A}=([Q], f)$ where $Q=2^{q}$ is a power of two with $q \geqslant 1$. We will denote by $\left(x^{\prime}(x, y), y^{\prime}(x, y)\right)$ the value of $f(x, y)$.

\section{Overview of the construction}

Due to space constraint, we will expose here the principle of the construction. The full description of the modules and of the attraction rule is given in appendix in Section B on page 20. 


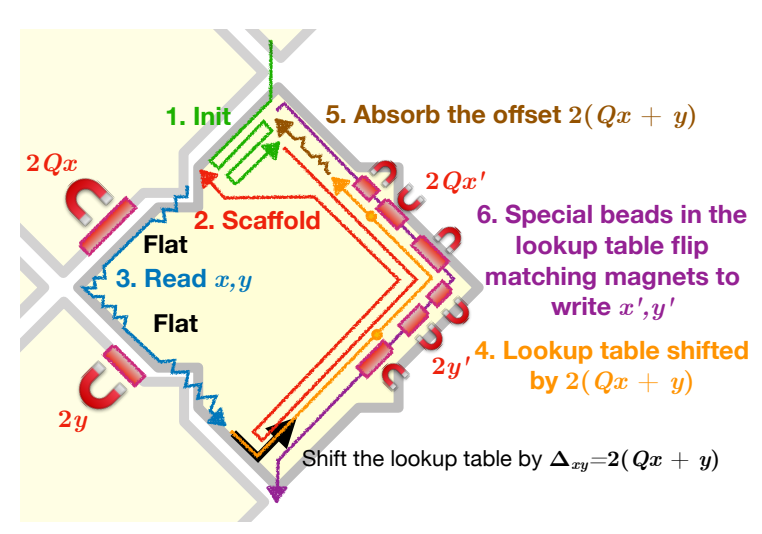

(a) Blueprint of the Oritatami cell design

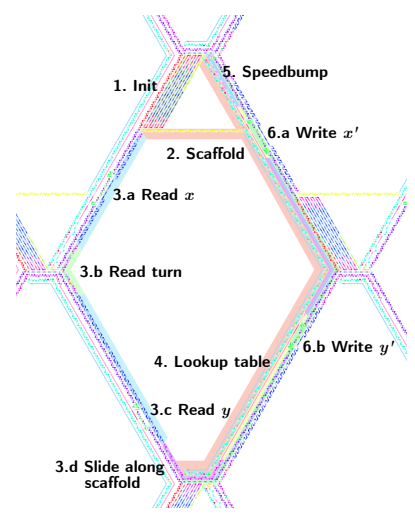

(b) Actual oritatami cell for $q=1(Q=2)$.

Fig. 1: The modules inside a cell: (Right) 1. Cell Init highlighted in yellow; 2. Scaffold in red; 3. Read in blue, green and purple; 4 . Lookup Table in yellow and violet; 5 . Speedbump in cyan; 6 . Write in green.

In our simulation, each cell of the simulated S2FA is represented by a supercell shaped as an hexagon with two short sides (N and S) of lengths 12 and 13 , and four long sides (NE, NW, SE and SW) of lengths $s$ and $s-1$ where $s=O\left(Q^{2} \log Q\right)$ (see Fig. 1b). The simulation proceeds by building one after the other the supercells simulating each of the cells of the simulated S2FA according to the up-down order given in Fig. 2. Each supercell is the result of the folding of exactly one period of the transcript. The period of transcript consists in the sequence of 6 modules, each of them achieving one specific task:

$$
\mathbf{D} \cdot \mathbf{S} \cdot \mathbf{R} \cdot \mathbf{L} \cdot \mathbf{S B} \cdot \mathbf{W}
$$

The modules. Their respective roles and positions inside the supercell are blueprinted in Fig. 1a. 耳 is responsible for extending the configuration by one supercell and reversing the up-down order at the end of the current column of supercells (see Fig. 10 on page 16). S has two roles: providing a scaffold along which the next modules will fold, and ensuring that the molecule "resynchronizes" (will be defined later) before $\mathbf{W}$ writes the two outputs $x^{\prime}$ and $y^{\prime}$ on the output sides. $\mathbf{R}$ is responsible for reading the value of the two inputs $x$ and $y$ and translating accordingly the lookup table of the simulated S2FA, encoded in the next module L. SB is responsible for "resynchronizing" the molecule along the scaffold, annihilating the translation of the lookup table induced by the reading of $x$ and $y$ by $\mathbf{R}$. Finally, $\mathbf{W}$ writes on the output sides of the supercell the values $x^{\prime}$ and $y^{\prime}$ dictated by the translated lookup table $\mathbf{L}$, and exits the supercell at the entrance of the next one.

Encoding $x$ and $y$. The values of $x$ and $y$ are encoded along the sides of the supercells using "magnetic flipping flaps" of total lengths $4 Q x$ and $4 y$ respec- 


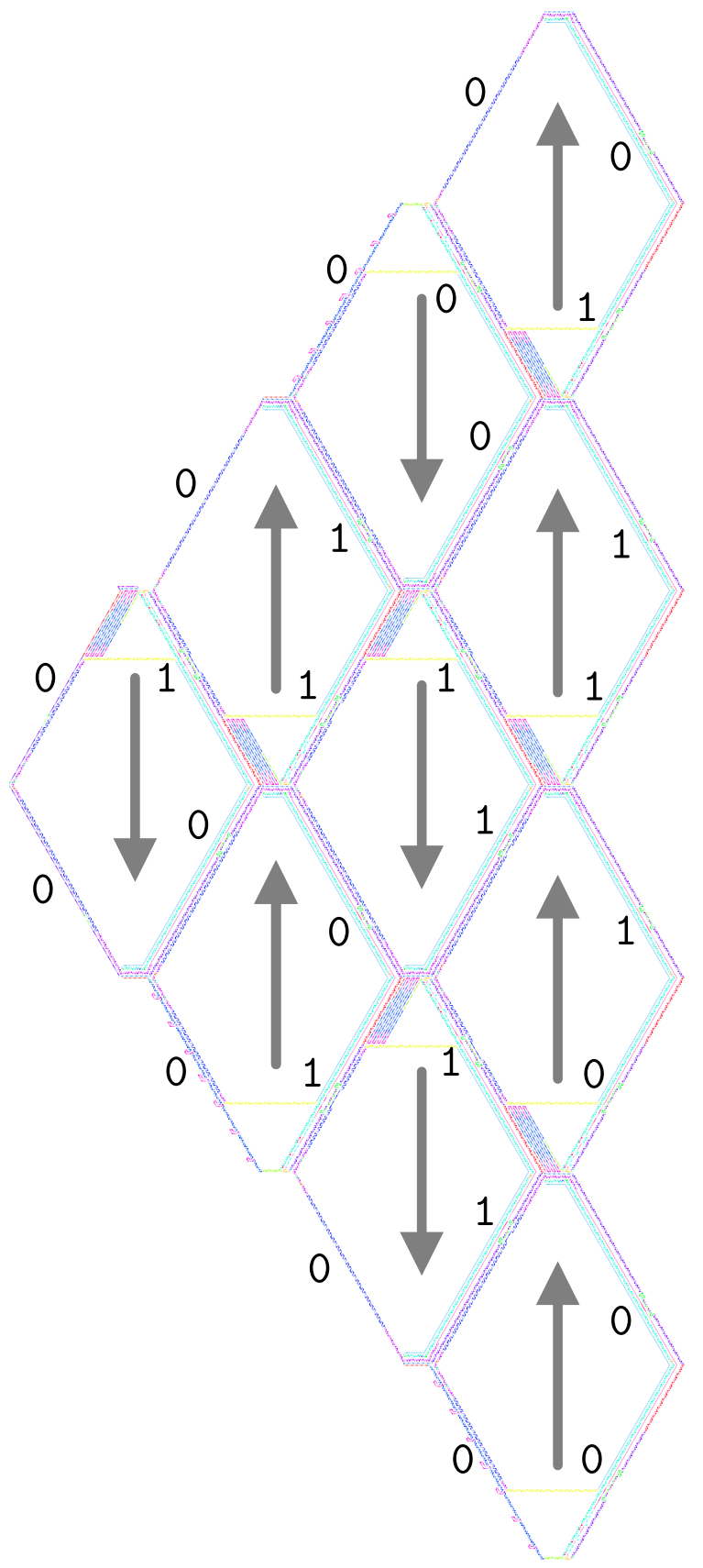

Fig. 2: The 10 first super-steps of the Oritatami simulation of the 2-states S2FA $(q=1): f(x, y)=(y+1 \bmod 2, x)$. 
tively, as schematically shown on Fig. 1a. When the read module $\mathbf{R}$ folds along the side of the neighboring supercells, it gets flatten by these magnetic flaps; this shifts the progression of the molecule forward by exactly half the lengths of the flaps. It follows that the module $\mathbf{R}$ completes its folding $\Delta_{x y}=2(Q x+y)$ beads further than it would in absence of the magnetic flaps. This, in turn, translates the position of the lookup table module $\mathbf{L}$ by $\Delta_{x y}$ along both output sides of the supercell, placing the entries corresponding to $x^{\prime}\left(\Delta_{x y}\right)=x^{\prime}(x, y)$ and $y^{\prime}\left(\Delta_{x y}\right)=y^{\prime}(x, y)$ in front of the flipping flaps of the module $\mathbf{W}$ to be folded next so that, when folded, the total magnetic length of the flipping flaps on each output side is $4 Q x^{\prime}$ and $4 y^{\prime}$ respectively (see Section 4 and Fig. 4 for details).

\section{Description of the key mechanisms}

Due to space constraints, we will focus on the new mechanisms involved in this construction. In particular, we will not discuss $\square$ because its behavior is just a direct translation of the Module G in [3] (see Section A.3 for details). $\mathbf{S}$ is simply hardcoded and only its key part will be discussed next in Section 4.2.

\subsection{Modules $R$, $L$ and $W$ : The read, lookup, write mechanism}

The previous section gave the principle of the interactions between these modules: the reading of $x$ and $y$ on the input sides consists for $\mathbf{R}$ in shifting the lookup table $\mathbf{L}$ by $\Delta_{x y}=2(Q x+y)$, which aligns the entries corresponding to $x^{\prime}(x, y)$ and $y^{\prime}(x, y)$ properly with the flaps of module $\mathbf{W}$ which writes the corresponding $x^{\prime}(x, y)$ and $y^{\prime}(x, y)$ on the $x^{\prime}$ - and $y^{\prime}$-output side respectively using the magnetic flaps as illustrated on Fig. 4. Let us start with Module L. Please refer to Fig. 3 for the alignment of the various parts involved.

Module $\mathbf{L}$. Each output $x^{\prime}(x, y)$ and $y^{\prime}(x, y)$ is encoded in binary into $q$ tables of $Q^{2}$ bits using bead types $\mathbf{Q 0}$ and $\mathbf{Q} 1$. The entry indexed $Q x+y$ in the $i$-th table for $x^{\prime}$ (resp. $\left.y^{\prime}\right)$ contains the value of the $i$-th bit of $x^{\prime}(x, y)$ (resp. $\left.x^{\prime}(x, y)\right)$. More precisely, if we write $x^{\prime}(x, y)=\sum_{i=0}^{q-1} x_{i}^{\prime}(x, y) 2^{i}$ in binary, the table for $x^{\prime}$ consists

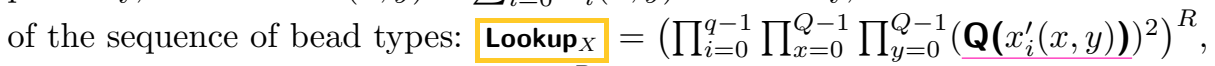
such that the bead types in $\operatorname{Lokup}_{X}{ }^{R}$ at indices $0,2 Q^{2}, \ldots,(q-1) 2 Q^{2}$ shifted by $\Delta_{x y}=2(Q x+y)$ are $\mathbf{Q}\left(b_{0}\right), \ldots, \mathbf{Q}\left(b_{q-1}\right)$ with $b_{i}=x_{i}^{\prime}(x, y)$, the $i$-th bit of $x^{\prime}(x, y)$. Lookup $Y$ is defined similarly.

Module $\mathbf{W}$ consists in a zigzag glider T0..7 that runs along the two output sides of the supercell, together with $q$ "magnetic flipping flaps", equally spaced by $2 Q^{2}$ beads on each output side (see Fig. 3): $q$ flaps of lengths $2^{0} Q, \ldots, 2^{q-1} Q$ on the $x^{\prime}$-output side and of lengths $2^{0}, \ldots, 2^{q-1}$ on the $y^{\prime}$-output side. We define a magnetic flipping flap of length $\ell$ as the bead type sequence: 


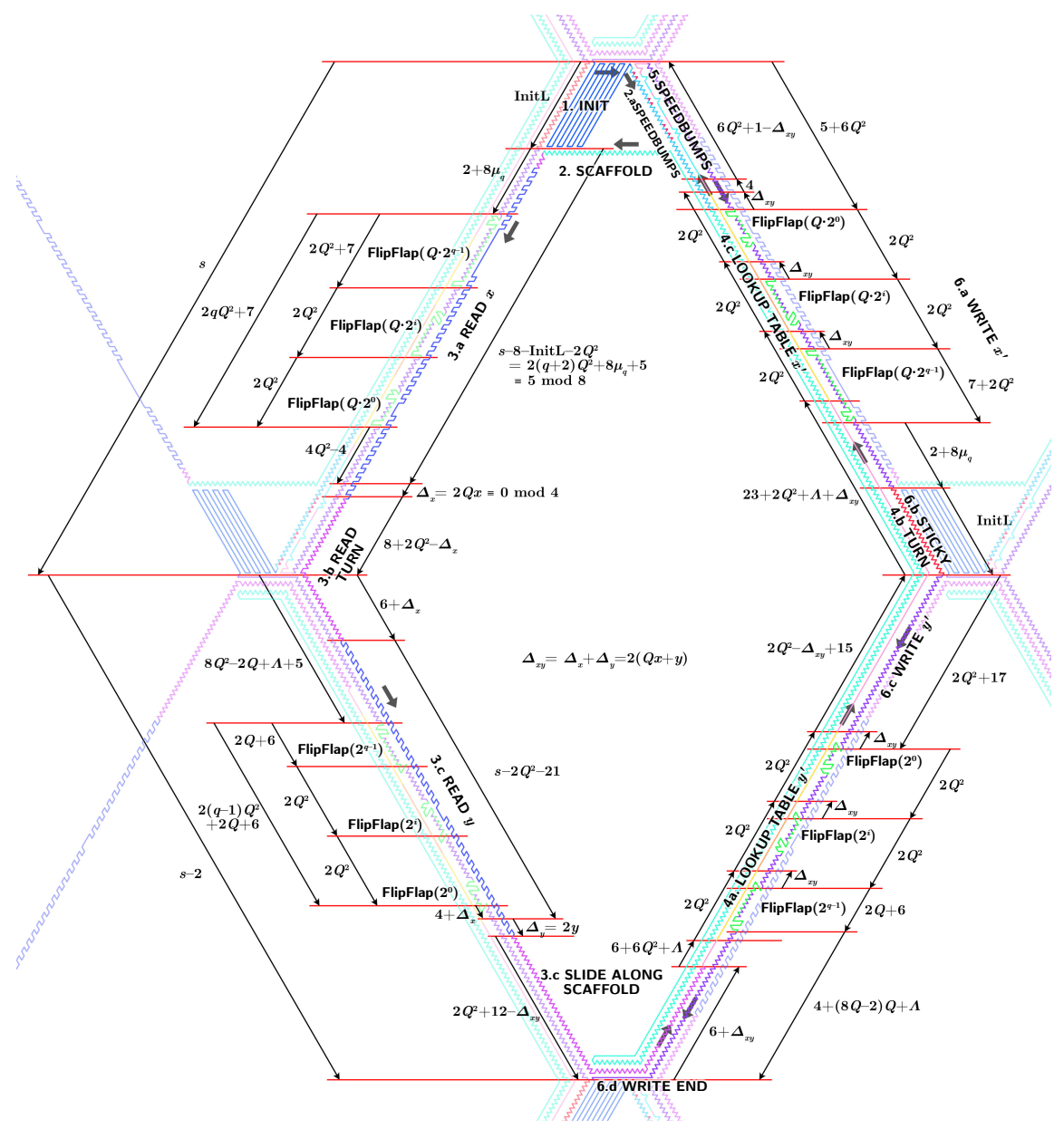

Fig. 3: Alignment of the various modules

Each flap is either activated (magnetic for $\mathbf{R}$ ) or deactivated (neutral for $\mathbf{R}$ ) depending on whether its "magnetic" beads P0..3 point outwards, towards the upcoming neighboring supercell, or inwards, towards the inside of the current supercell been folded (see Fig. 4). Now, thanks to the alignment of the modules (see Fig. 3), the $i$-th flap of $\mathbf{W}$ starts folding in front of the entries $\Delta_{x y}+2 i Q^{2}$ of the lookup table on each output side, that is in front of the pair of beads $\mathbf{Q} \mathbf{0}^{2}$ or $\mathbf{Q} \mathbf{1}^{2}$ corresponding to the $i$-th bit of the value to write on this side. Now, a flap folds outwards (is activated) by defaults, unless its initial bead $\mathbf{U} 5$ is attracted by a pair of beads Q0 corresponding to a bit set to 0 . It follows that the $i$-th flap of $\mathbf{W}$ on each side is activated if and only if the $i$-th of the output is 1 ; and as it is of length $2^{i} Q$ and $2^{i}$ for the $x^{\prime}$ - and $y^{\prime}$-output side respectively, the 
total numbers of magnetic beads are $4 Q x^{\prime}(x, y)$ and $4 y^{\prime}(x, y)$ on each $x^{\prime}$ - and $y^{\prime}$-output side respectively!

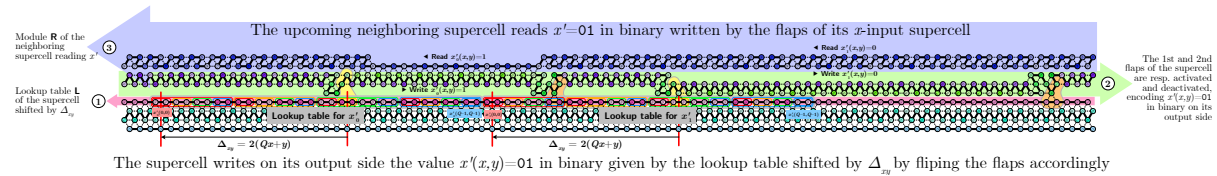

(a) 1. Lookup table, 2. write and 3. read modules on the $x^{\prime}$-output side when offset $\Delta_{x y}=2 \times 9$ (i.e. $\left.(x, y)=(2,1)\right)$ The supercell writes on its output side the value of $y^{\prime}(x, y)=10$ in binary given by the lookup table shifted by $\Delta_{x y}$

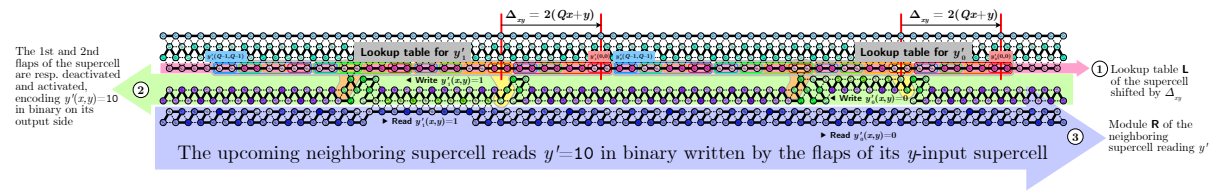

(b) 1. Lookup table, 2. write and 3. read modules on the $y^{\prime}$-output side when offset $\Delta_{x y}=2 \times 4$ (i.e. $\left.(x, y)=(1,0)\right)$

Fig. 4: Illustration of the border between two neighboring supercells: Interactions of 1 . the lookup table and 2 . the write modules within a supercell and 3 . the read module of the upcoming neighboring supercell, when $Q=4(q=2)$. The lookup tables for each bit follow each other and are $2 Q^{2}$ beads long each ( 2 beads per bit). The write module folds into $q=2$ flipping flaps on each output side of lengths $4 \times 2^{0}$ and $4 \times 2^{1}$ on the $x^{\prime}$-output side and $2^{0}$ and $2^{1}$ on the $y^{\prime}$-output side. We have highlighted in yellow the folding of bead U5 which decides of the orientation of each flap (in-/out-wards). We have highlighted in orange the folding of bead $\mathbf{U 6}$ which is attracted by all bead types Q0..2 and thus restores the orientation of the write glider to defaults after each flap.

Module $\mathbf{R}$. We are now ready to conclude this mechanism by observing that the read module $\mathbf{R}$ folds along the write modules of the two neighboring input supercell, and that it gets flatten each time it folds along an activated flap (see Fig. 3 and 4), which extends its length by half the number of magnetic beads P0..3 of the flap. It follows that the end of its folding is shifted forward by $\overline{(4 Q x}+4 y) / 2=\Delta_{x y}$, which in turn shifts forward the lookup table module $\mathbf{L}$ by $\Delta_{x y}$ as claimed! Refer to Fig. 6 for a complete view of the folding of $\mathbf{R}$.

The full description of the modules $\mathbf{R}, \mathbf{L}$ and $\mathbf{W}$ may be found in appendix in Sections B.4, B.5, and B.7.

\subsection{Modules SB and S: resynchronization using speedbumps}

In order for the period of the transcript to end precisely at the exit of the supercell, whatever inputs $x$ and $y$ were read by the read module $\mathbf{R}$, we need to 
absorb the $\Delta_{x y}$ offset. Precisely, we need to absorb it before the write module $\mathbf{W}$ folds to ensure that it is properly aligned with the shifted lookup table. This is the role of the speedbump module SB. Its behavior is illustrated on Fig. 5 .

This mechanism involves two modules: the scaffold module $\mathbf{S}$, which contains the speedbumps (consisting in alternation of red beads $\mathbf{1 0 . . 3}$ and blue beads E0..3) at the top of its NE corner (assuming the supercell is in the downwards orientation); the speedbump module SB which consists in a matching alternation of red beads $\mathbf{Q} 2$ and blue beads R0..1.

Lemma 1 (speedbump). When a red-blue sequence $\gamma=\mathbf{Q 2}{ }^{4 k-1} \mathbf{R 1}(\mathbf{R 0} . . \mathbf{1})^{4 k}$ folds from right to left over a blue-red-blue seed left-to-right sequence $\sigma=$ $(\mathbf{E} 2 \mathrm{E} 4 \mathrm{E6E0})^{k}(\mathbf{I 1 . . 3 1 0})^{k}(\mathbf{E} 2 \mathrm{E} 4 \mathrm{E6E0})^{2 k}$ starting from the $\Delta$-th rightmost position

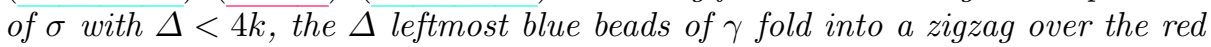
beads of $\sigma$, and the folding of $\gamma$ ends at the $\lfloor\Delta / 2\rfloor$ rightmost position of the left red segment of $\sigma$, as shown in Fig. $5 b$.

Proof. There are two cases to consider depending on the parity of the offset $\Delta$ of the molecule when entering a red speedbump. In both cases, at the exit of the red speedbump, the new offset is $\lfloor\Delta / 2\rfloor$.

Corollary 1. When the folding of the speedbump module $\mathbf{S B}$ completes, the offset $\Delta_{x y}$ is totally absorbed.

Proof. Note the maximum offset when the speedbump module $\mathbf{S B}$ starts to fold is $\Delta=2\left(Q^{2}-1\right)$ corresponding to reading input $(x, y)=(Q-1, Q-1)$. The matching exponentially decreasing alternation of blue and red regions from $2^{2 q}$ to 4 in $\mathbf{S}$ and SB (see descriptions in Sections B.3 and B.6) ensures that the offset is divided by 2 until it reaches 0 , absorbing the total offset.

This concludes the construction. Refer to Fig. 5 for an illustration of the process.

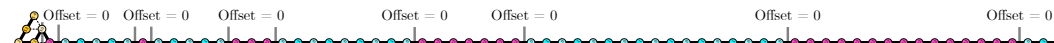

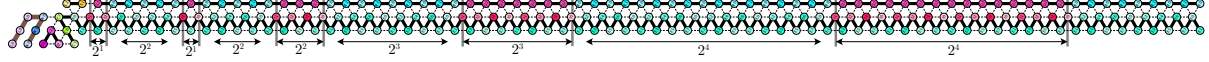

(a) Synchronized speedbump: all the top red parts are inside the red speedbumps

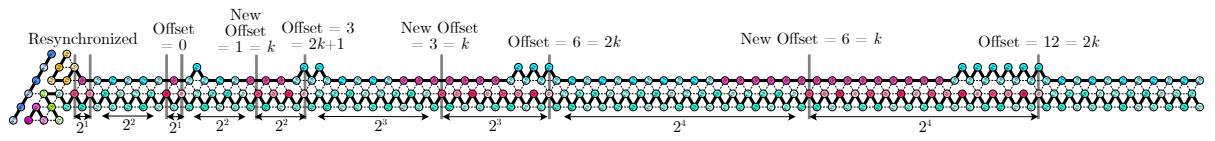

(b) Synchronizing speedbump: each time the blue part of the molecule passes over a red speedbump, the offset $\Delta$ is divided by 2 .

Fig. 5: Speedbumps decrease exponentially the offset of the molecule folding on top (going from right to left) until it vanishes. 


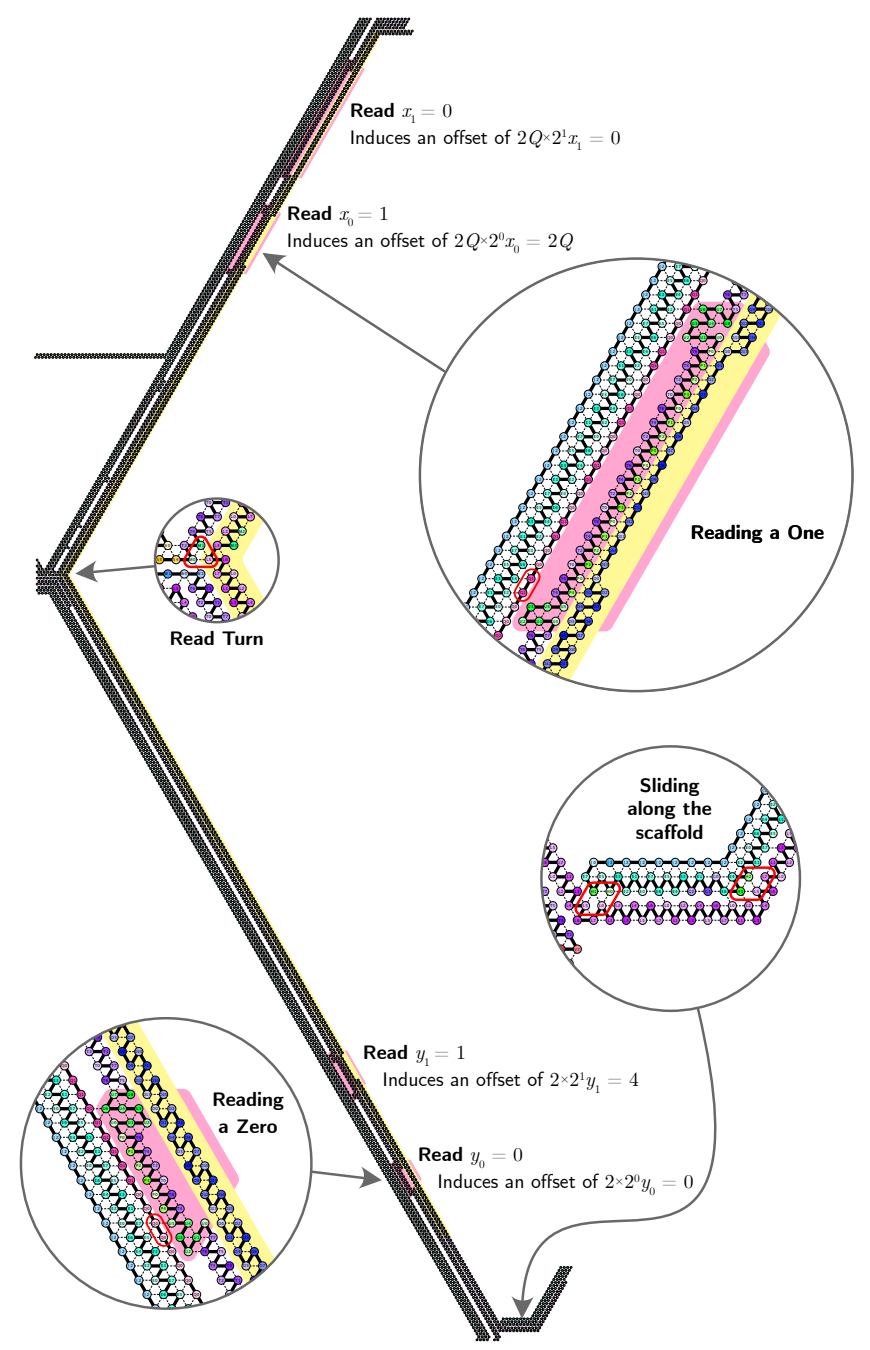

Fig. 6: Read module consists of 4 parts: ReadX, Turn, ReadY, Slide along the scaffold. ReadX and ReadY get flatten each time it passes along an outward write magnetic flap (encoding a 1). This shifts the molecule by the length of the corresponding flap. The following part of the molecule is then shifted overall by $\Delta_{x y}=2(Q x+y)$. This shift allows then to align the entry corresponding to $(x, y)$ of the lookup table of each side with the upcoming write modules. The two glider-based parts "Turn" and "Slide along" are only there to ensure that after reading each input, the molecule turns at the expected position, regardless of the offset. 


\subsection{Correctness of the folding}

Finally, the correctness of the folding is obtained by choosing the size $s$ of the supercell so that all modules are properly aligned. This is ensured by enforcing the position of every pattern in every module modulo 8 in the supercell as exposed in Fig. 3 and detailled in the appendix section.

\section{References}

1. Cook, M.: Universality in elementary cellular automata. Complex Systems 15(1) (2004)

2. Demaine, E., Hendricks, J., Olsen, M., Patitz, M., Rogers, T., Schabanel, N., Seki, S., Thomas, H.: Know when to fold 'em: Self-assembly of shapes by folding in oritatami. In: DNA. LNCS, vol. 11145, pp. 19-36 (2018)

3. Geary, C., Meunier, P.E., Schabanel, N., Seki, S.: Proving the Turing universality of oritatami cotranscriptional folding. In: ISAAC. LIPIcs, vol. 123, pp. 23:1-23:13 (2018)

4. Geary, C., Meunier, P.E., Schabanel, N., Seki, S.: Oritatami: A computational model for molecular co-transcriptional folding. Int. J. Mol. Sci. 20(9), 2259 (2019), preliminary version published in MFCS 2016

5. Geary, C., Rothemund, P.W.K., Andersen, E.S.: A single-stranded architecture for cotranscriptional folding of RNA nanostructures. Science 345, 799-804 (2014)

6. Han, Y.S., Kim, H.: Ruleset optimization on isomorphic oritatami systems. Theor. Comput. Sci. 785, 128-139 (2019)

7. Masuda, Y., Seki, S., Ubukata, Y.: Towards the algorithmic molecular self-assembly of fractals by cotranscriptional folding. In: CIAA. LNCS, vol. 10977, pp. 261-273. Springer (2018)

8. Ollinger, N.: Two-states bilinear intrinsically universal cellular automata. In: FCT. LNCS, vol. 2138, pp. 396-399. Springer (2001)

9. Ota, M., Seki, S.: Ruleset design problems for oritatami systems. Theor. Comput. Sci. 671, 26-35 (2017)

10. Padilla, J.E., Patitz, M.J., Schweller, R.T., Seeman, N.C., Summers, S.M., Zhong, $\mathrm{X}$.: Asynchronous signal passing for tile self-assembly: Fuel efficient computation and efficient assembly of shapes. Int. J. Found. Comput. Sci. 25(4), 459-488 (2014)

11. Padilla, J.E., Sha, R., Kristiansen, M., Chen, J., Jonoska, N., Seeman, N.C.: A signal-passing DNA strand exchange mechanism for active self-assembly of DNA nanostructures. Angew. Chem. Int. Edit. 54(20), 5939-5942 (2015)

12. Rogers, T.A., Seki, S.: Oritatami system; a survey and the impossibility of simple simulation at small delays. Fund. Inform. 154(1-4), 359-372 (2017)

13. Schabanel, N.: iOS CAOS simulator, hub.darcs.net/nikao0o0o0/CA0SSimulator

14. Theyssier, G.: Automates Cellulaires: un Modèle de Complexités. Ph.D. thesis, École Normale Supérieure de Lyon (2005)

15. Woods, D., Chen, H., Goodfriend, S., Dabby, N., Winfree, E., Yin, P.: Active selfassembly of algorithmic shapes and patterns in polylogarithmic time. In: ITCS. pp. 353-354 (2013)

16. Woods, D., Doty, D., Myhrvold, C., Hui, J., Zhou, F., Yin, P., Winfree, E.: Diverse and robust molecular algorithms using reprogrammable DNA self-assembly. Nature 567, 366-372 (2019)

17. Woods, D., Neary, T.: On the time complexity of 2-tag systems and small universal Turing machines. In: FOCS. pp. 439-448 (2006) 


\section{A Omitted Contents}

\section{A.1 Figure from Section 1}

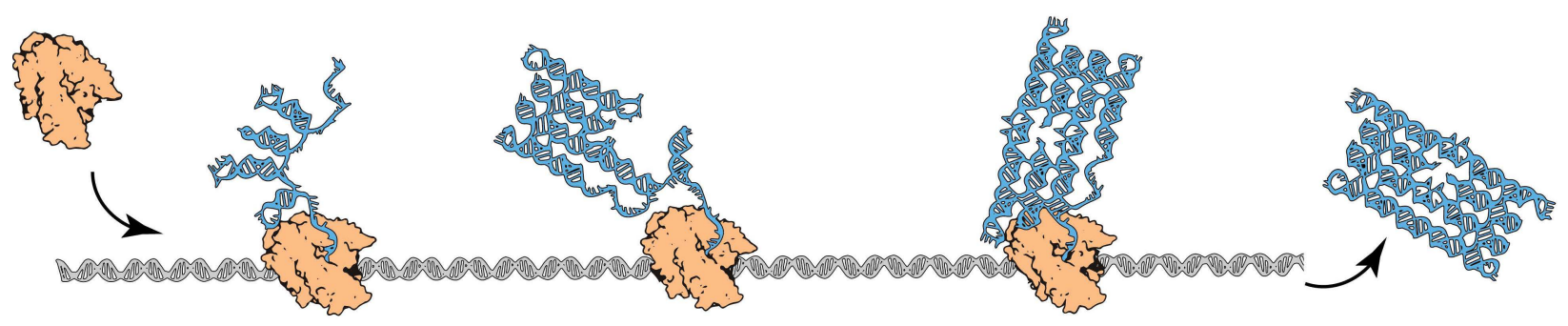

Fig. 7: RNA origami [5]: An RNA transcript (colored in blue) folds into a specific RNA rectangular tile structure in-vitro while being synthesized by RNA polymerase (orange).

\section{A.2 Figures from Section 3}

\section{A.3 Omitted Mechanisms}

Module D: Cell initialization This module is directly inspired from the Module G in [3]. It has two possible conformations as illustrated on Fig. 10:

- The compact conformation (Fig. 10a) consists in 8 switchbacks of height InitL. This conformation is triggered by the fact that if there is a neighboring cells, the beads $\mathbf{V} \mathbf{1}, \mathbf{V} \mathbf{3}, \mathbf{V} \mathbf{5}, \mathbf{V} \mathbf{7}$ on its side will attract П's first beads $\mathbf{A} \mathbf{0}$ and $\mathbf{A} \mathbf{1}$ forcing the Module to adopt the compact conformation.

- The extended conformation (Fig. 10b) consists in a glider of length $s$ which will extend the configuration by one supercell. This conformation is triggered by the absence of neighboring cell, its prefix beads $\mathbf{A 0}$ and $\mathbf{A} \mathbf{1}$ fold then along the beads $\mathbf{W} \mathbf{0}$ and $\mathbf{W} \mathbf{1}$, let the subsequent beads to adopt their spontaneous glider shape ending with a straight glider $(\mathbf{C 0 . . 7})^{2}$ miroring upside-down the growth of the next configuration and thus miroring upside-down the filling order of the next supercells.

Module S: scaffold Module S is simply hardcoded and adopts only one single conformation as illustrated in Fig. 15 on page 26.

Module R This module, or more precisely its transcript, consists of four parts $\operatorname{Read}_{X}, \operatorname{Read}_{T u r n}, \operatorname{Read}_{Y}$, and $\operatorname{Read}_{\text {Slide }}$ in this order, as illustrated on Fig. 6. $\boldsymbol{R e a d}_{X}$ and $\operatorname{Read}_{Y}$ are a repetition of 00..15, which folds into a special glider of width 8 and height 2 except in front of an outward flap from the write module, which is a part of $\mathbf{W}$, along which it gets flattened, creating an offset (see Figure 11). 


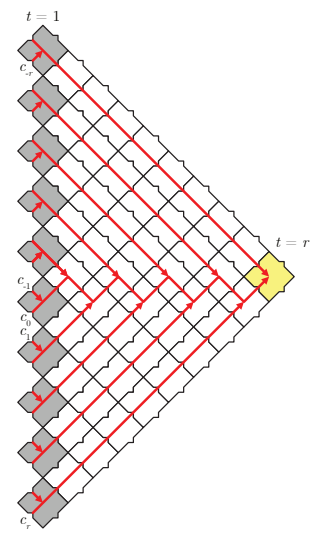

(a) CA simulated by 2FA

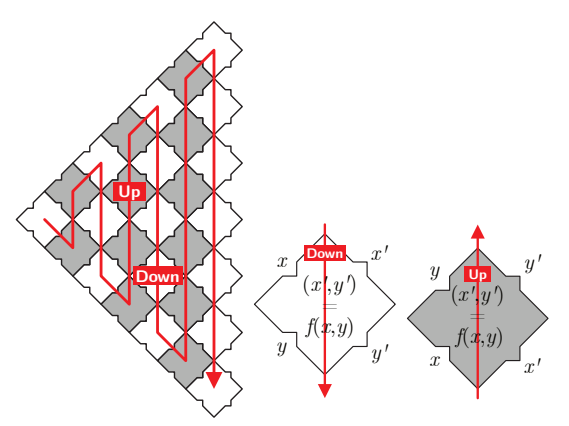

(b) Sweeping 2-fan-in 2-fan-out automata

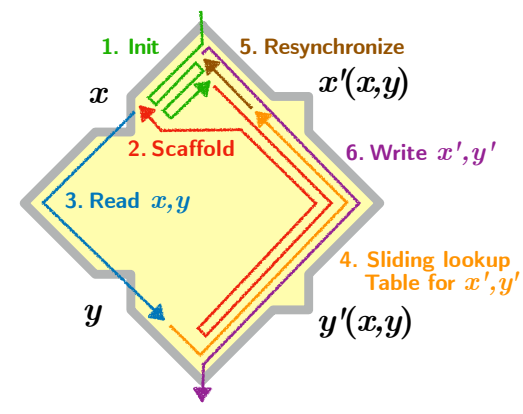

(c) Oritatami cell design

Fig. 8: Blueprint for intrinsic simulation of 1D cellular automata by Oritatami systems 


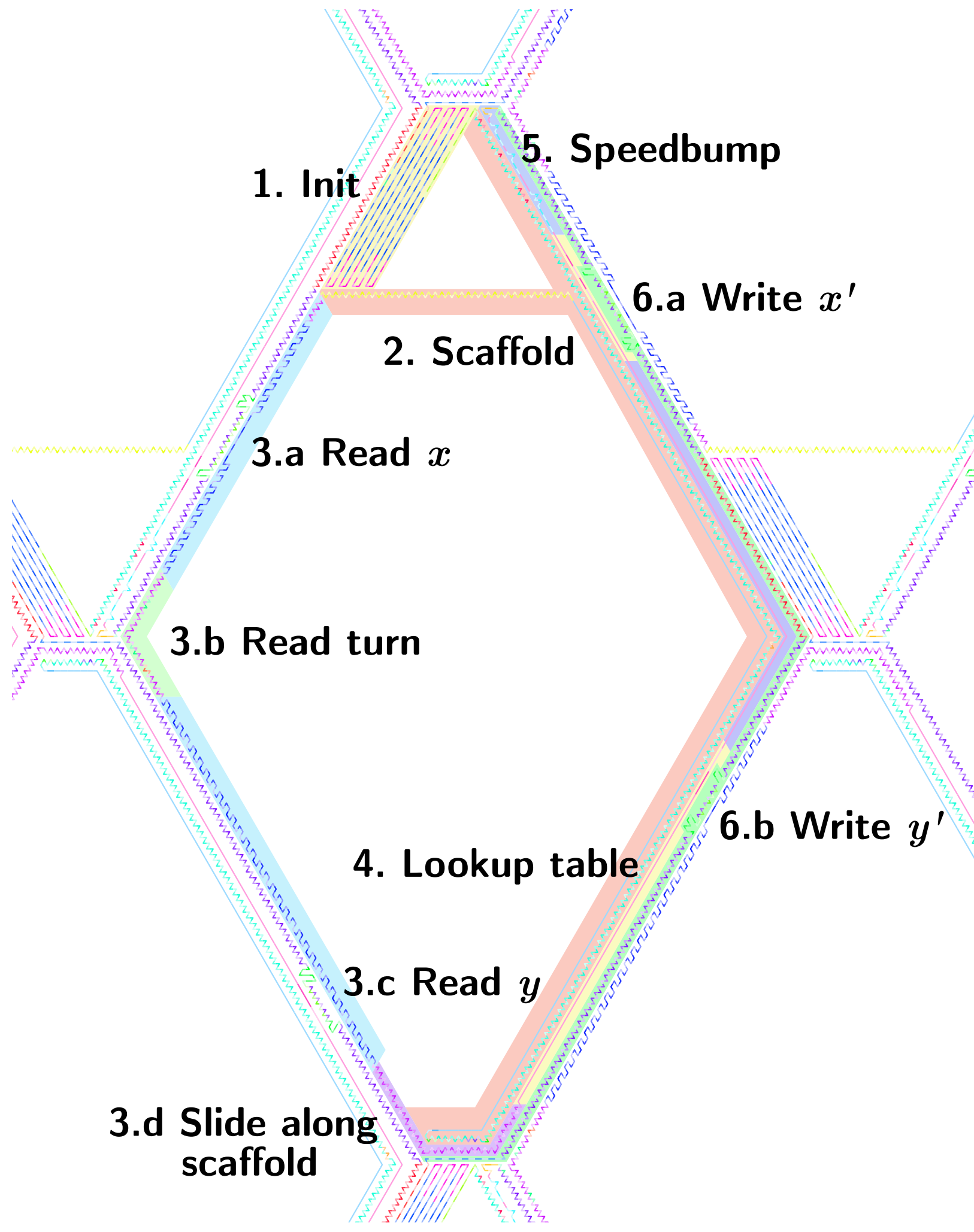

Fig. 9: The modules inside a supercell for a 2-states S2FA $(q=1)$ : 1. Cell Init highlighted in yellow; 2. Scaffold in red; 3 . Read in blue, green and purple; 4. Lookup Table in yellow and violet; 5 . Speedbump in cyan; 6 . Write in green. 


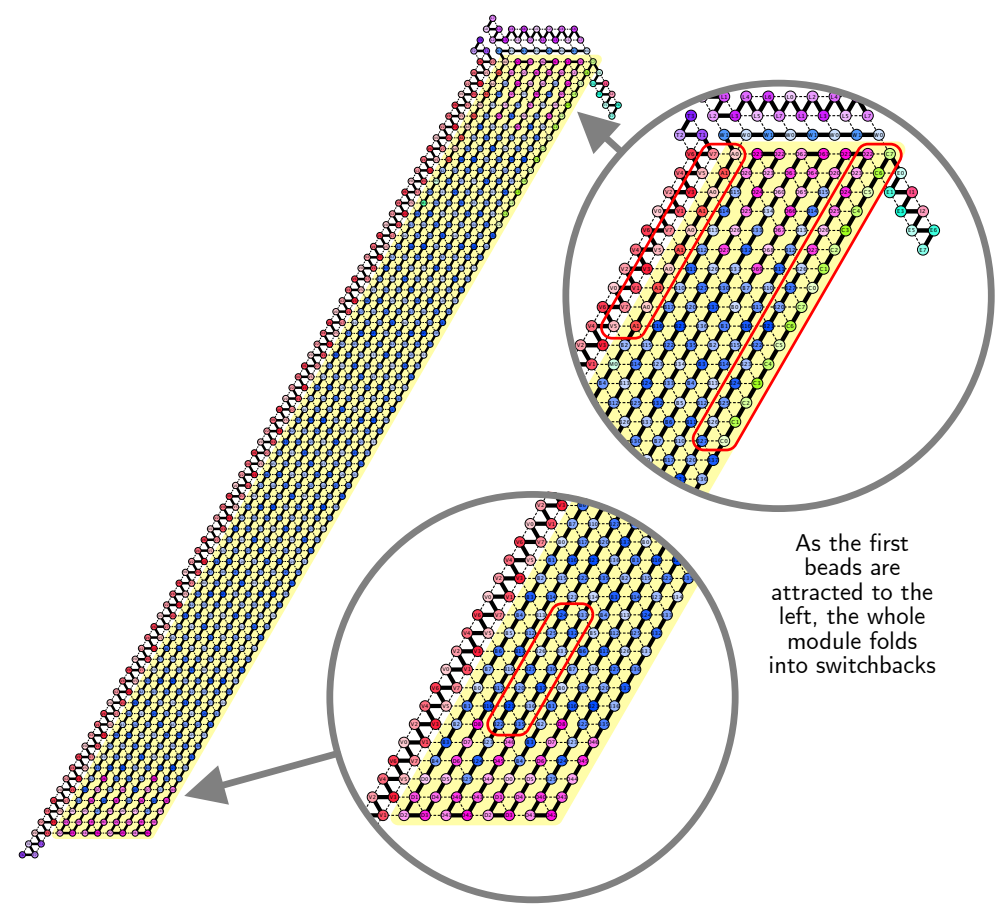

(a) The switchback form adopted by the Cell Init module when the southwest neighbor is there.

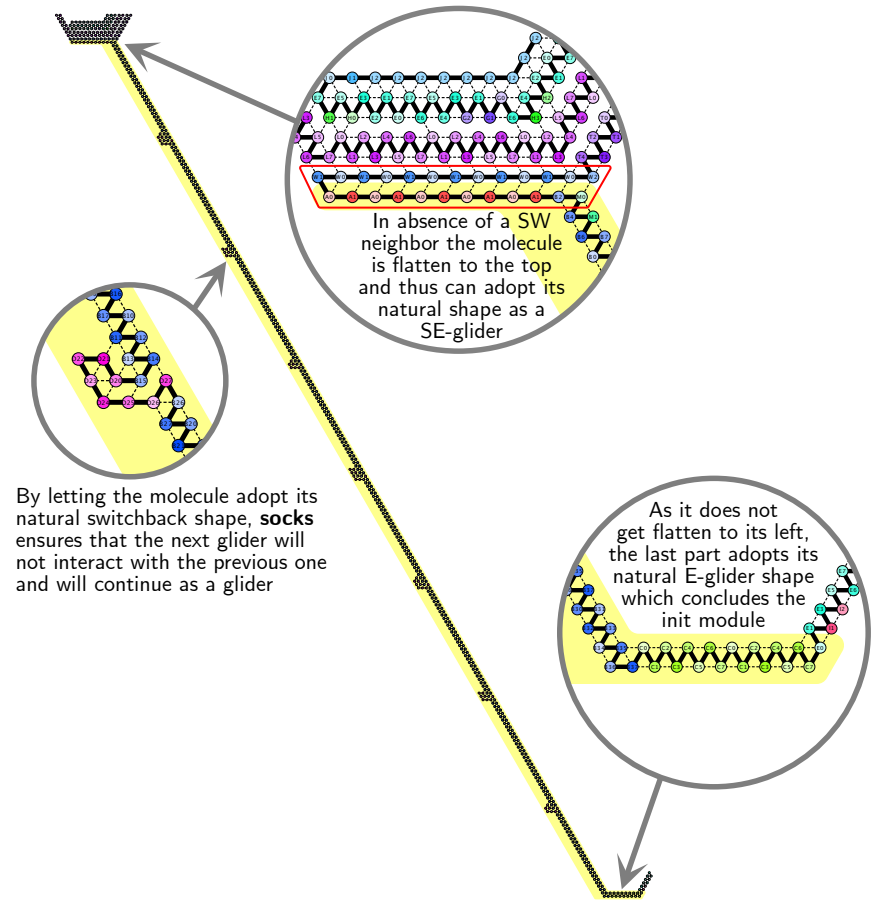

(b) The glider form adopted by the Cell Init module when there is no southwest neighbor. It creates the missing side (with a Zero input) and flips the sweeping direction of the simulated S2FA.

Fig. 10: The two bricks for the Cell Init module 1 . 

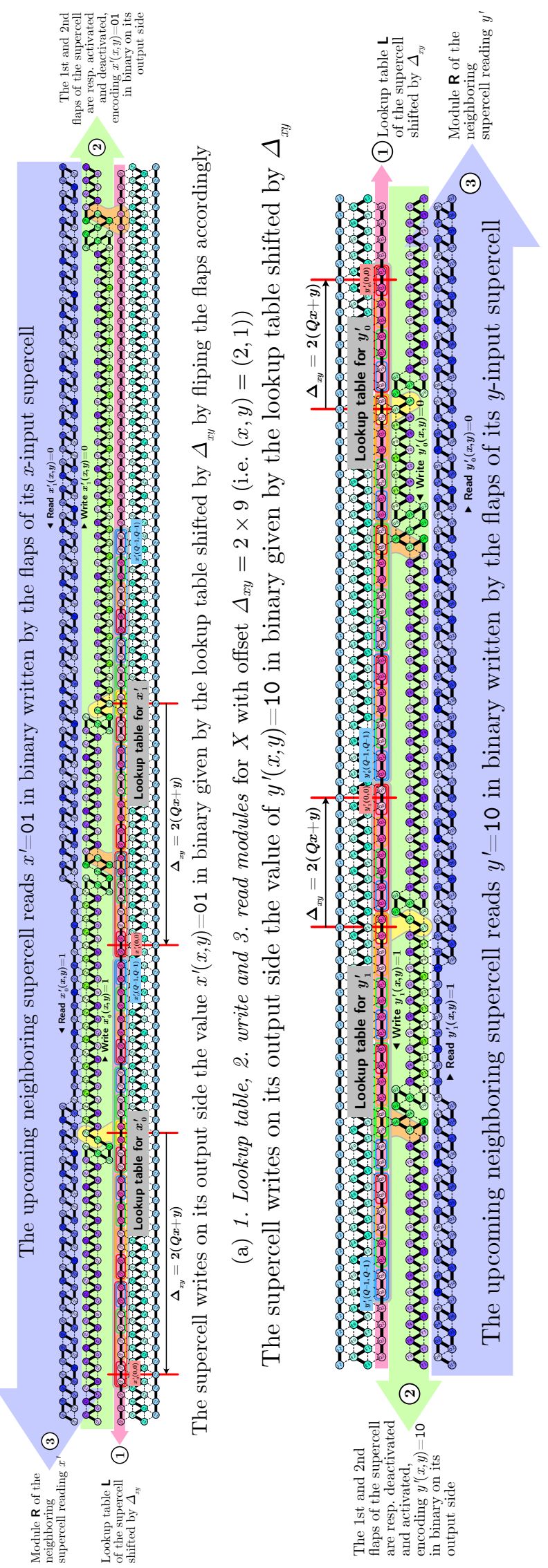

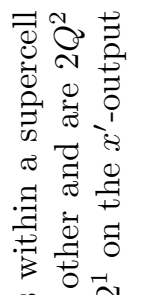

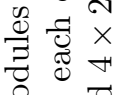

몸

â.

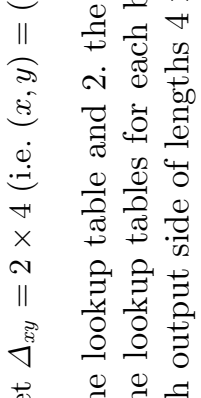

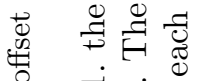

考宁宗

خ

항

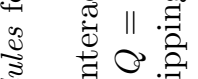

है

ङ

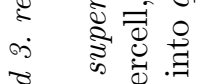

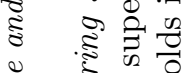

告

จ

ङ

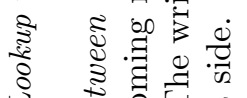

政

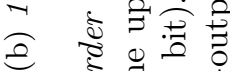

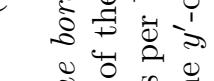

$\approx$

뉴요

(ㄷ)

ㅎํำ 근

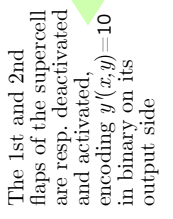

密芯

胥

$\ddot{\exists} \dot{0}$ 的

它政 


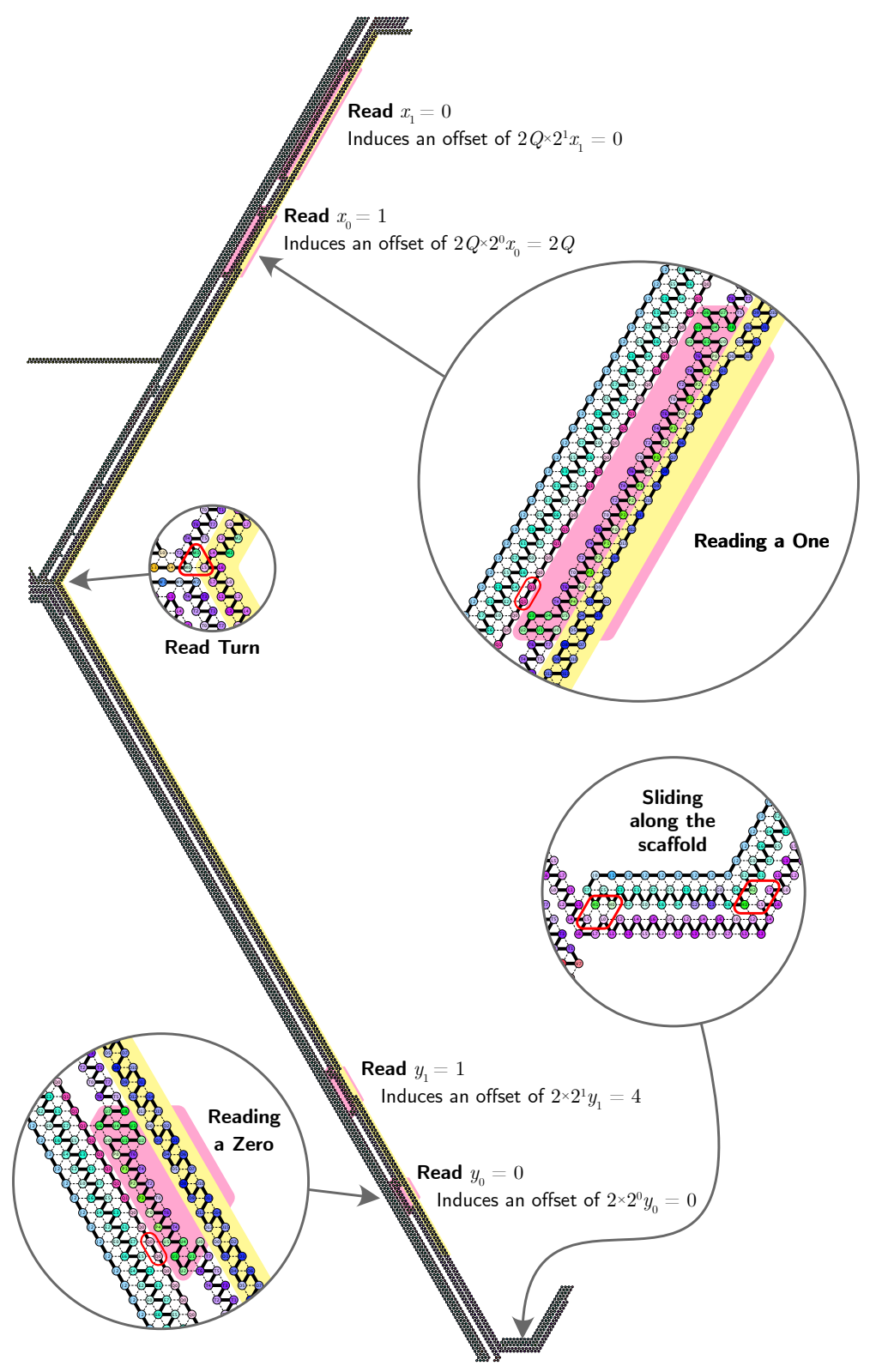

Fig. 12: Read module consists of 4 parts: ReadX, Turn, ReadY, Slide along the scaffold. ReadX and ReadY get flatten each time it passes along an outward write magnetic flap (encoding a 1). This shifts the molecule by the length of the corresponding flap. The following part of the molecule is then shifted overall by $\Delta_{x y}=2(Q x+y)$. This shift allows then to align the entry corresponding to $(x, y)$ of the lookup table of each side with the upcoming write modules. The two glider-based parts "Turn" and "Slide along" are only there to ensure that after reading each input, the molecule turns at the expected position, regardless of the offset. 


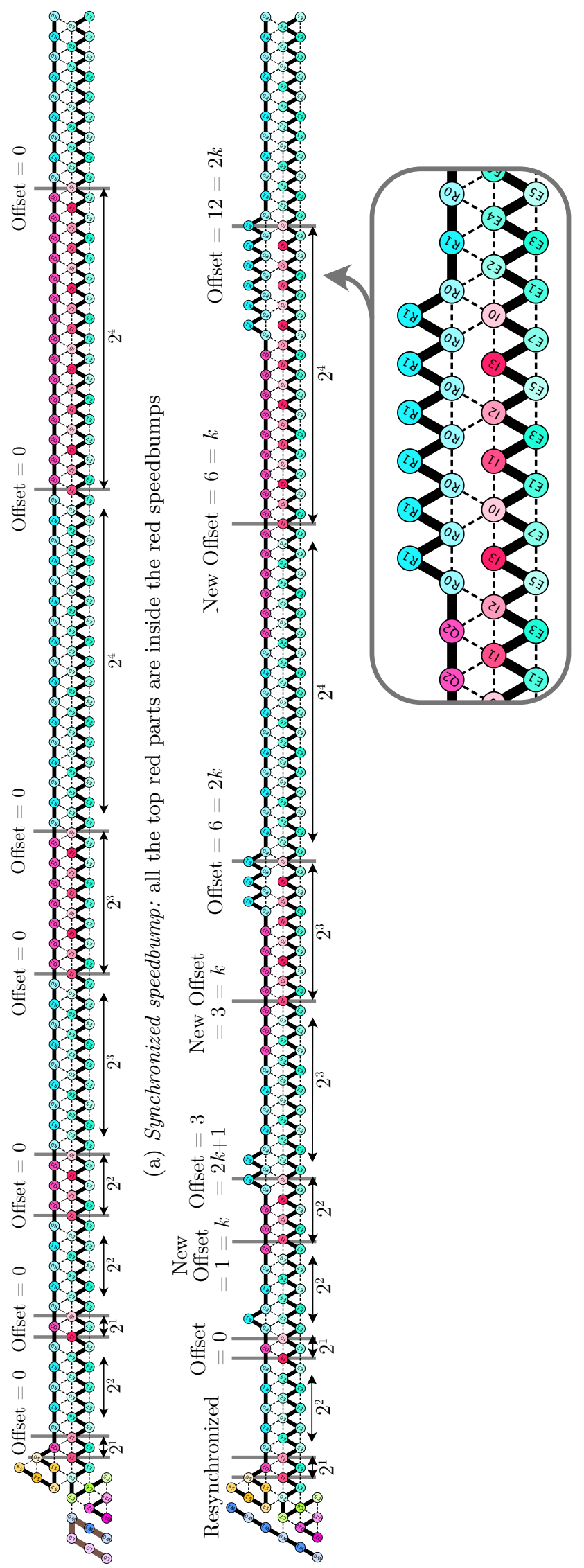

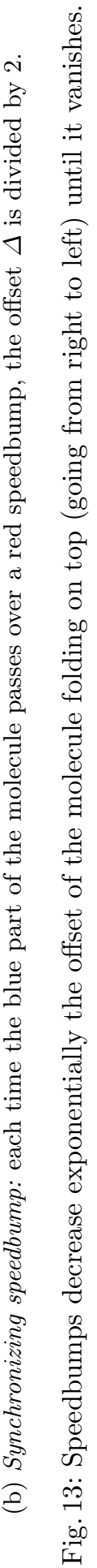




\section{B Full description of intrinsic CA simulator}

Variables for convenience. In order to simplify the following description, let us introduce the following variables, which correspond to key geometrical parameters:

$$
\begin{aligned}
Q & =2^{q}, \text { for some integer } q \geqslant 1 \\
\mu_{q} & =2 q \bmod 3 \\
\lambda & =\frac{2 q Q^{2}+8 \mu_{q}}{3} \\
\Lambda & =\frac{2 q Q^{2}+32 \mu_{q}}{3}=\lambda+8 \mu_{q} \\
\text { InitL } & =\text { SmallestMultipleOf8GreaterOrEqualTo }\left(2 Q^{2}+16+\left\lceil\frac{2 q Q^{2}}{3}\right\rceil\right) \\
& =\frac{(3+q) \cdot 2 Q^{2}+8 \mu_{q}}{3}+16=16+2 Q^{2}+\lambda \\
s & =4 \text { InitL }-35 \\
\text { InitX } & =3 Q^{2} / 2 \\
\text { InitY } & =Q^{2} / 2+4
\end{aligned}
$$

Let us prove (1) InitL $=\frac{(3+q) \cdot 2 Q^{2}+8 \mu_{q}}{3}+16$. Indeed, as $2 \equiv-1 \bmod 3$ and $Q=2^{q} \equiv(-1)^{q} \bmod 3$, we have: $2 q Q^{2}+8 \mu_{q} \equiv-q(-1)^{2 q}-(-q) \equiv 0 \bmod 3$. It follows that $\left(2 q Q^{2}+8 \mu_{q}\right) / 3$ is an integer. Furthermore, as $2 q Q^{2}$ and $8(2 q \bmod 3)$ are both multiple of 8 , this integer is necessarily a multiple of 8 (as 3 and 8 are relatively prime). Finally, as $8 \mu_{q} / 3<8$, the claimed expression is indeed the smallest multiple of 8 as wanted. This implies that $\lambda$ and $\Lambda$ are integers, indeed: $2 q Q^{2}+8 \mu_{q} \equiv 2 q Q^{2}+32 \mu_{q} \equiv(2 q-2 q) \equiv 0 \bmod 3$.

The following equality will be useful for ensuring the proper alignments of the various parts of the molecule as it folds:

$$
\begin{aligned}
Q & \equiv 0 \bmod 2 \\
\lambda \equiv \Lambda & \equiv 0 \bmod 8 \\
\text { InitL } & \equiv 0 \bmod 8 \\
s & \equiv 29 \bmod 32 \equiv 5 \bmod 8 \\
\text { InitX } & \equiv 0 \bmod 6 \\
\text { InitY } & \equiv 0 \bmod 2
\end{aligned}
$$

Notations for describing bead type sequences. We denote by $\underline{\mathbf{A} i . . j}$ the sequence of bead types $\underline{\mathbf{A} i}, \underline{\mathbf{A}(i+1)}, \ldots$, $\mathbf{A} j$ if $j \geqslant i$, and $\underline{\mathbf{A} i}, \mathbf{A}(i-1), \ldots, \mathbf{A} j$ if $j<i$.

For two finite bead type sequences $u$ and $v$, we denote their concatenation by $u \cdot v$. Given a sequence $u$, we write $u\left\langle\left\langle a_{1} @ i_{1}, \ldots, a_{k} @ i_{k}\right\rangle\right\rangle$ for the sequence $w$ obtained by replacing the bead indexed by $i_{j}$ in $u$ with $a_{j}$ for all $1 \leqslant j \leqslant k$, that is,

$$
w_{i}= \begin{cases}a_{j} & \text { if } i=i_{j} \text { for some } j \\ u_{i} & \text { otherwise }\end{cases}
$$

Notations for describing conformations. Given an infinite sequence of directions $\left(d_{i}\right) \in\left\{\begin{array}{l}\mathrm{NE} \\ \nearrow\end{array}, \overrightarrow{\mathrm{E}}, \mathrm{SE}, \stackrel{\swarrow}{\mathrm{SW}}, \overleftarrow{\mathrm{W}}, \nwarrow^{\mathrm{NW}}\right\}$ and a finite bead type sequence $b \in B^{*}$, we denote by Conformation $(b, d)$ the conformation $b_{0} d_{0} b_{1} d_{1} \cdots d_{|b|-2} b_{|b|-1}$, which maps $b$ along the path $d$. In the description of bricks, the following func- 
tions shall turn out to be useful:

$$
\begin{aligned}
& \text { NE-path }(b)=\text { Conformation }\left(b,\left({ }_{\nearrow}^{\mathrm{NE}}\right)^{\infty}\right) \text {, } \\
& \text { E-path }(b)=\text { Conformation }\left(b,(\overrightarrow{\mathrm{E}})^{\infty}\right) \text {, } \\
& \text { SE-path }(b)=\text { Conformation }\left(b,(\mathrm{SE})^{\infty}\right) \text {, } \\
& \text { SW-path }(b)=\text { Conformation }\left(b,\left(\swarrow_{\mathrm{SW}}\right)^{\infty}\right) \text {, } \\
& \text { W-path }(b)=\text { Conformation }\left(b,(\overleftarrow{\mathrm{W}})^{\infty}\right), \\
& \text { NW-path }(b)=\text { Conformation }\left(b,\left(\aleph^{\mathrm{NW}}\right)^{\infty}\right) \text {. }
\end{aligned}
$$

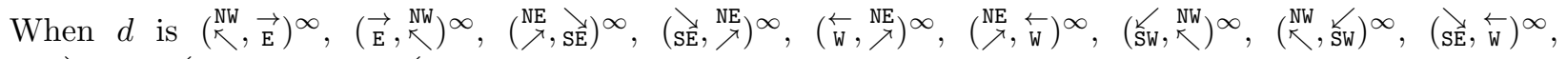
$(\overleftarrow{\mathrm{W}}, \underset{\mathrm{SE}}{\searrow})^{\infty},(\stackrel{\mathrm{SW}}{\swarrow}, \overrightarrow{\mathrm{E}})^{\infty}$, or $(\overrightarrow{\mathrm{E}}, \underset{\mathrm{SW}}{\swarrow})^{\infty}$, we use $\operatorname{Glider}(b, d)$ as an alias of Conformation $(b, d)$ because with such $d$ Conformation $(b, d)$ becomes a directional zigzag structure that reminds of the glider in the study of cellular automata. Functions $\circlearrowright_{\pi / 3}$ and $\circlearrowleft_{\pi / 3}$ rotate a path or conformation given as an argument by degree $\pi / 3$ clockwise and counterclockwise, respectively.

\section{B.1 Periodic transcript}

The transcript is periodic and its period is semantically divided into six modules as

$$
\mathbf{D} \cdot \mathbf{S} \cdot \mathbf{R} \cdot \mathbf{L} \cdot \mathbf{S B} \cdot \mathbf{W} .
$$

These modules shall be described fully one by one below. Summing their lengths, which shall be analyzed also there, results in $71 \mathrm{InitL}+10 q-526=O\left(Q^{2} \log Q\right)$ as the length of this period. 


\section{B.2 Cell Init}

Transcript length: 8 InitL

\# of bricks: 2

\# of bead types: 78

Bead types: A0, A1, B0..7, B10..17, B20..27, B30..37, C0..7, D0..8, D20..27, D40..46,

D60..69, M0, M1

Transcript.

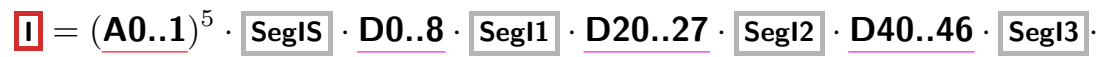

$$
\begin{aligned}
& \text { D60..69 · Segl0 } \cdot \text { D0..8 } \cdot \text { Segl1 } \cdot \text { D20..27 } \cdot \text { Segl2 } \cdot \text { D40..46 } \cdot \text { SegIE } \cdot(C 0 . .7)^{2} \text {, }
\end{aligned}
$$

where

$$
\begin{aligned}
& \text { SegIS }=\underline{B 2} \cdot \text { M0 } \cdot \underline{\text { B4 }} \cdot \text { M1 } \cdot \underline{\text { B6..7 }} \cdot(\underline{B 0 . .7})^{\mathrm{InitL} / 8-3} \cdot \underline{\text { B0..4 }} \\
& \text { Seg10 }=\underline{\text { B7 }} \cdot(\underline{B 0 . .7})^{\text {InitL } / 8-2} \cdot \underline{\text { B0..4 }} \\
& \text { Segl1 }=\text { B16..17 } \cdot(\text { B10..17 })^{\operatorname{InitL} / 8-2} \cdot \text { B10..15 } \\
& \text { Seg12 }=\text { B26..27 } \cdot(\text { B20..27 })^{\text {InitL } / 8-2} \cdot \text { B20..25 } \\
& \text { Seg13 }=\underline{\text { B35..37 }} \cdot(\underline{B 30 . .37})^{\mathrm{InitL} / 8-2} \cdot \text { B30..34 } \\
& \text { SegIE }=\text { B35...37 } \cdot(\text { B30..37 })^{\text {InitL } / 8-3}
\end{aligned}
$$

Bricks. Module 1 expects two conformations (bricks), which are shown in Figure 10:

The switchback brick Isb is a parallelogram of width 8 and height InitL consisting of 8 switchbacks in the $\swarrow_{\text {SW }}^{\swarrow}$ and ${ }_{\nearrow}^{\mathrm{NE}}$ directions:

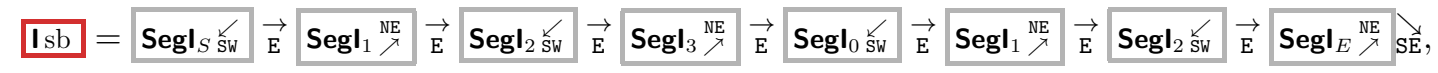

where

$$
\begin{aligned}
& \text { Segl } \left._{S \text { SW }}=S W-\text { path }(\underline{(\mathbf{A 0 . . 1}})^{5} \cdot \operatorname{SegIS} \cdot \underline{\text { D0..2 }}\right) \text {, } \\
& \text { SegI }_{0} \mathrm{SW}_{\mathrm{W}}=\text { SW-path }(\underline{\text { D63..69 }} \cdot \operatorname{Seg} 10 \cdot \underline{\text { D0..2 }}) \text {, }
\end{aligned}
$$

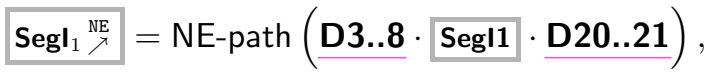

$$
\begin{aligned}
& \text { Segl }_{2} \stackrel{\text { SW }}{=}=\text { SW-path }(\text { D22..27 } \cdot \text { Segl2 } \cdot \text { D40..41), } \\
& \operatorname{Segl}_{3}{ }_{3}^{\mathrm{NE}}=\text { NE-path }(\text { D42..46 } \cdot \text { Segl3 } \cdot \text { D60..62), } \\
& \operatorname{Segl}_{E} \stackrel{\text { NE }}{Y}=\text { NE-path }\left(\text { D42..46 } \cdot \text { SegIE } \cdot(\text { C0..7 })^{2}\right) \text {. }
\end{aligned}
$$


The glider brick Iexp consists of a 2-thick SĔ-path creating the missing side of length $s$ of the new cell to be created at the bottom of the configuration. The newly created side reads input state 0 :

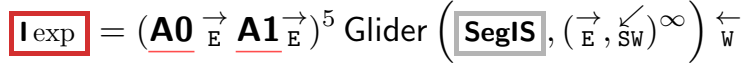

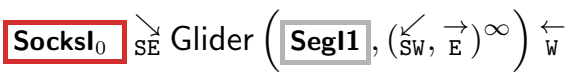

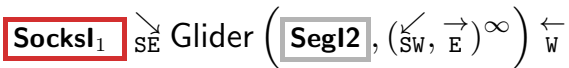

$$
\begin{aligned}
& \text { SocksI }_{2} \text { SE Glider }\left(\text { Segl3 },(\underset{\text { SW }}{\swarrow}, \vec{E})^{\infty}\right) \overleftarrow{W} \\
& \text { SocksI }_{3} \text { SE Glider }\left(\text { Segl0 },(\underset{\text { SW }}{\swarrow}, \vec{E})^{\infty}\right) \overleftarrow{W} \\
& \text { SocksI }{ }_{0} \text { SE Glider }\left(\text { Segl1 },(\underset{\text { SW }}{\swarrow}, \vec{E})^{\infty}\right) \overleftarrow{W}
\end{aligned}
$$

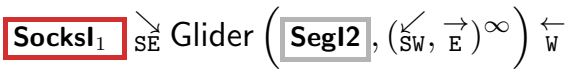

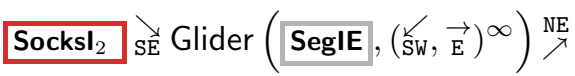

$$
\begin{aligned}
& \operatorname{Glider}\left((\mathbf{C 0} . .7)^{2},\left(\underset{\mathrm{SE}}{\searrow}, \mathrm{NE}^{\mathrm{NE}}\right)^{\infty}\right)
\end{aligned}
$$

where

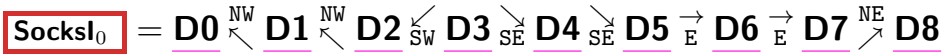

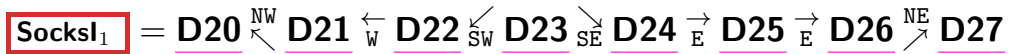

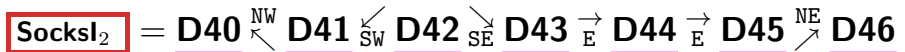

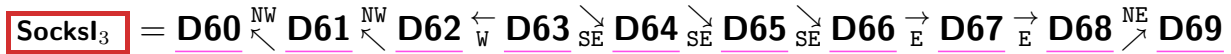

Fact 1 The vector from the origin of $\overrightarrow{\operatorname{lexp}}$ to the origin of the succeeding $\mathbf{S}$ is $(s-2) \overrightarrow{\mathrm{se}}+20 \overrightarrow{\mathrm{e}}$, and from the origin of $\mathbf{l s b}$ to the succeeding $\mathbf{S}$ is $8 \overrightarrow{\mathrm{e}}$.

Subrule. Figure 14 presents the subrule for the interactions between the bead types in $\square$ and all the other modules. 


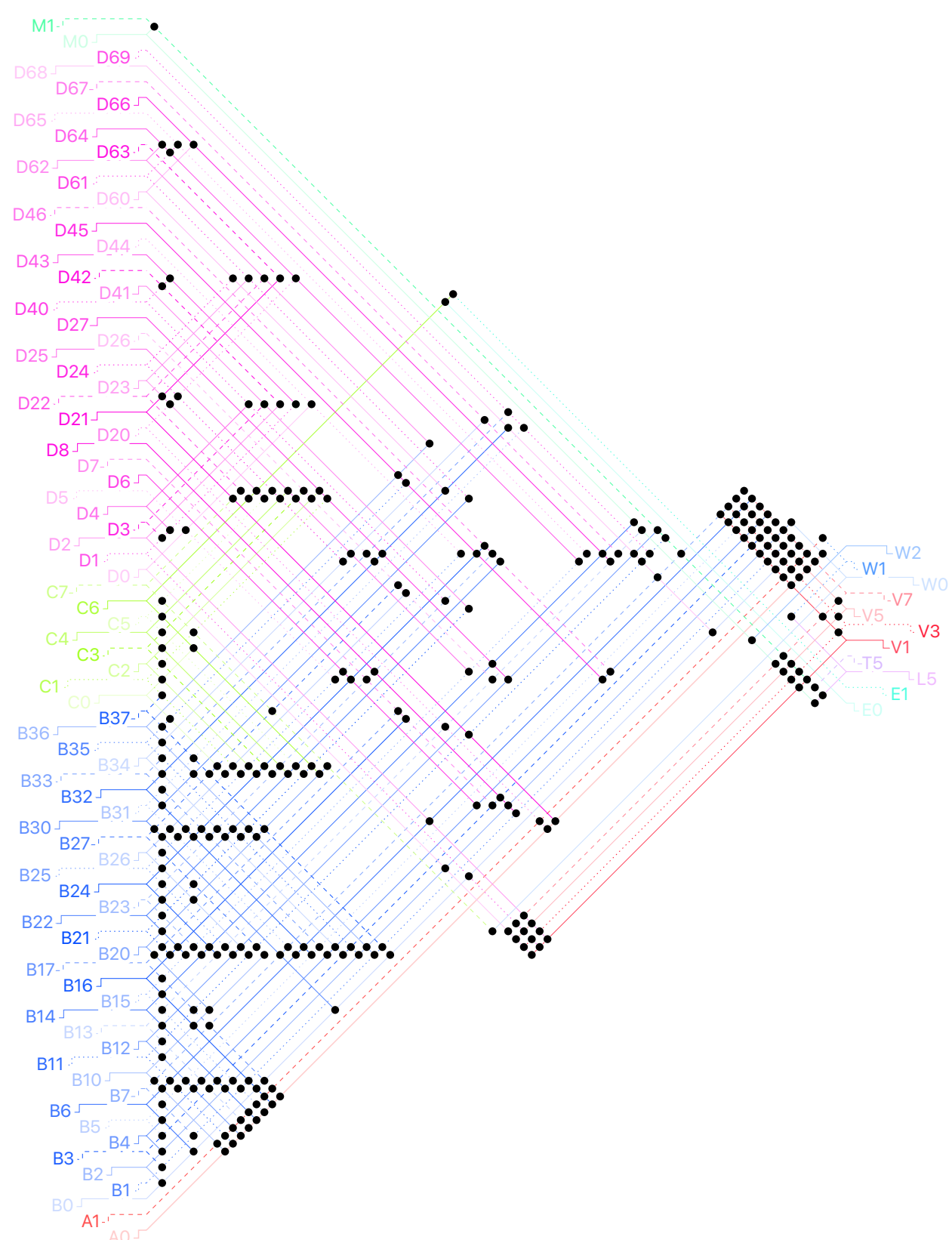

Fig. 14: Subrule for Module 1 


\section{B.3 Scaffold}

Transcript length: $\quad$ InitL $+6 s+15$

\# of bricks: 1

\# of bead types: 33

Bead types: E0..7, F0..2, G0..2, H0..3, 10..3, J0..2, K0..7

Transcript. The transcript of this module is

$$
\mathbf{S}=\text { ScaffoldG }_{S B} \cdot \text { ScaffoldG }_{X} \cdot \text { ScaffoldG }_{Y} \cdot \text { ScaffoldG }_{U} \cdot \text { ScaffoldG }_{S T} \cdot \text { ScaffoldG }_{L} \text {, }
$$

where

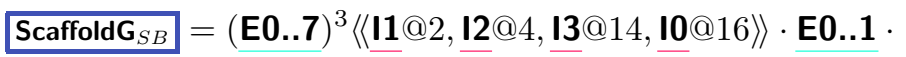

$$
\begin{aligned}
& \prod_{i=1}^{2 q-1}(\underline{\mathbf{I 1}} \cdot \underline{\mathrm{E} 3} \cdot \underline{\mathbf{1 2}} \cdot \underline{\mathrm{E} 5} \cdot \underline{\mathbf{I 3}} \cdot \underline{\mathrm{E} 7} \cdot \underline{\mathbf{I 0}} \cdot \underline{\mathrm{E} 1})^{2^{i-1}}(\underline{\mathrm{E} 2 . .7} \cdot \underline{\mathrm{E} 0 . .1})^{2^{i}} \\
& \text { ScaffoldG }_{X}=\mathbf{E 2} . \mathbf{. 7} \cdot(\mathbf{E} \mathbf{0 . . 7}) \overbrace{(s-1) / 4-3 Q^{2} / 2-1}^{6+Q^{2} / 2+\lambda} \text {, } \\
& \text { ScaffoldG }_{Y}=\text { F0..1 } \cdot \text { E2 } \cdot \text { F2 } \cdot \text { E4..7 } \cdot(E 0 . .7)^{(s-5) / 4-2} \cdot \text { E0..2 } \cdot \text { H2 } \cdot \text { E4 } \cdot \text { H3 } \cdot \text { E6, } \\
& \mathrm{ScaffoldG}_{U}=\mathrm{G0..1} \cdot \mathrm{E1} \cdot \mathrm{G2} \cdot \mathrm{E3} . .7 \cdot \mathrm{E0..3} \cdot \mathrm{H0} \cdot \mathrm{E5} \cdot \mathrm{H1} \cdot \mathrm{E7} \text {, } \\
& \text { ScaffoldG }_{S T}=\mathbf{J 0 . . 1} \cdot \mathbf{J} \mathbf{2}^{2 s-\text { InitL }-3} \text {, } \\
& \text { ScaffoldG }_{L}=\text { K4...7 } \cdot(\mathbf{K O} . .7)^{\text {InitL } / 4+1} \text {. }
\end{aligned}
$$

Note that the lengths of Scaffold $_{S B}$, ScaffoldG $\mathbf{G}_{X}$ and Scaffold $_{Y}$ are $12 Q^{2}+2$, $54+8 \lambda+4 Q^{2}=2(s-1)-\left(12 Q^{2}+2\right)$ and $2(s-5)-1$, respectively.

Brick. This module always folds into the following brick:

$$
\begin{aligned}
& \mathbf{S !}=\text { Glider }\left(\text { ScaffoldG }_{S B} \cdot \text { ScaffoldG }_{X},(\stackrel{\mathrm{SW}}{\swarrow}, \overrightarrow{\mathrm{E}})^{\infty}\right) \overleftarrow{\mathrm{W}} \text { Glider }\left(\text { ScaffoldG }_{Y},(\stackrel{\searrow \mathrm{SE}}{\overleftarrow{\mathrm{W}}})^{\infty}\right)^{\mathrm{NW}}
\end{aligned}
$$

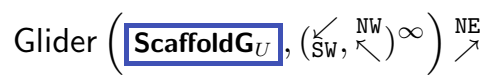

$$
\begin{aligned}
& \text { E-path }\left(\underline{\mathbf{J} 0} \cdot \underline{\mathbf{J 1}} \cdot \underline{\mathbf{J}}^{7}\right){ }^{\mathrm{NE}} \mathrm{N} \text { NE-path }\left(\underline{\mathbf{J}}^{s-7}\right){ }^{\mathrm{NW}} \mathrm{NW} \text {-path }\left(\underline{\mathbf{J}}^{s-\mathrm{InitL}-3}\right) \stackrel{\mathrm{NW}}{\nwarrow} \\
& \text { Glider }\left(\text { ScaffoldG }_{L},(\stackrel{\mathrm{NW}}{\nwarrow}, \underset{\mathrm{SW}}{\swarrow})^{\infty}\right) \text {, }
\end{aligned}
$$

which is illustrated in Figure 15 for $q=2$.

Fact 2 The vector from the beginning of $\mathbf{S !}$ to that of the succeeding $\mathbf{R}$ is $\operatorname{InitL} \overrightarrow{\mathrm{sw}}-8 \overrightarrow{\mathrm{e}}$.

Subrule. Figure 16 presents the subrule for the interactions between the beads of $\mathbf{S}$ and those of the other modules. 


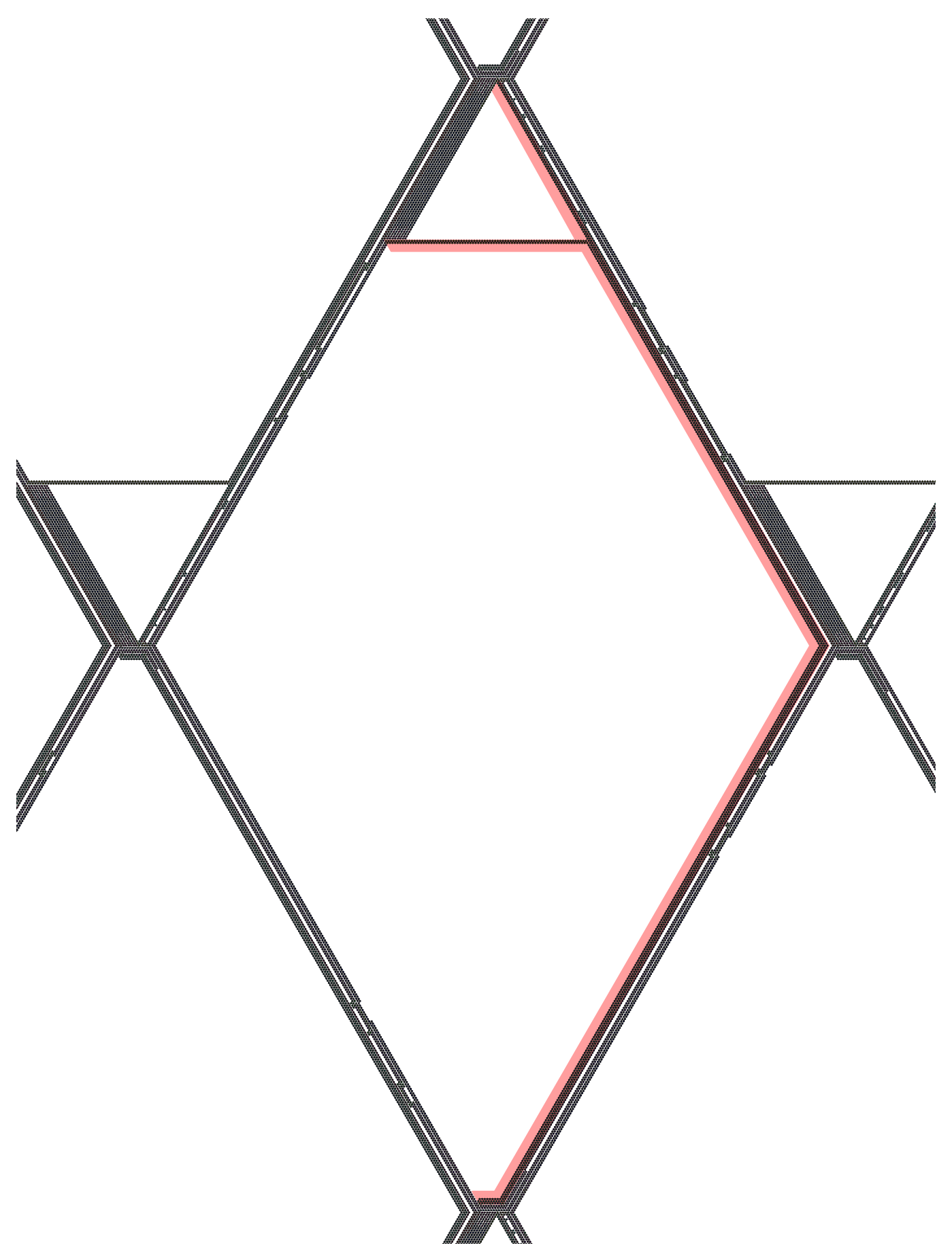

Fig. 15: The scaffold (highlighted in red) for $q=2$. 


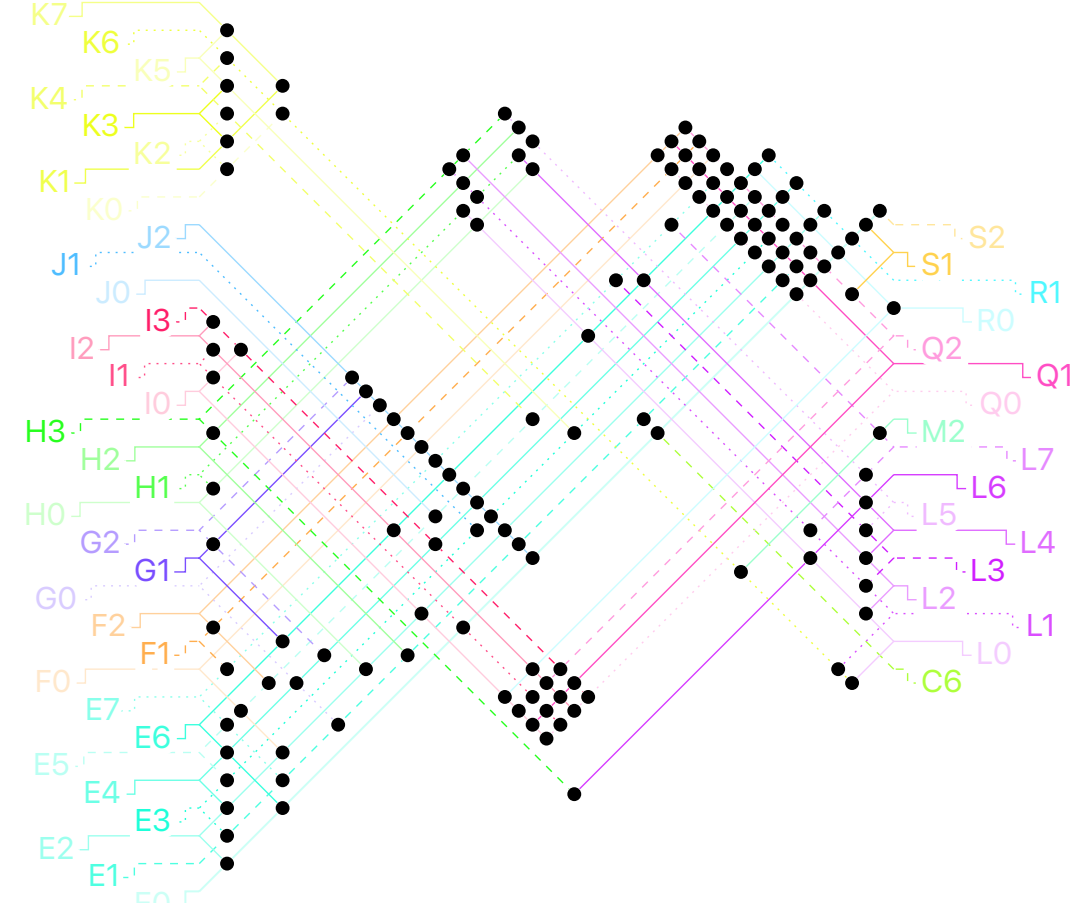

Fig. 16: Subrule for Module S. 


\section{B.4 Read}

Transcript length: $4 s-2$ InitL +18

\# of bricks: $Q^{2}$

\# of bead types: 29

Bead types L0..7, M2, M3, N0..2, 00..15.

Transcript. The transcript of this module is

$$
\mathbf{R}=\operatorname{Read}_{X} \cdot \operatorname{Read}_{T u r n} \cdot \operatorname{Read}_{Y} \cdot \operatorname{Read}_{\text {Slide }} \text {, }
$$

where

$$
\begin{aligned}
& \operatorname{Read}_{X}=\underline{\mathbf{L 0 . . 7}} \cdot(\underline{\mathbf{O 0} . .15})^{\left(s-5-\operatorname{InitL}-2 Q^{2}\right) / 8-1} \\
& \text { Read }_{\text {Turn }}=(\text { L0..7 } \cdot(\underline{\mathbf{L O} . .7}\langle\langle\mathbf{M} \mathbf{2} @ 1, \underline{\mathbf{M} 3} @ 3\rangle\rangle))^{Q^{2} / 4+1} \cdot \underline{\mathbf{N} 0 . .2} \cdot \underline{\mathbf{L O} . .7} \\
& \operatorname{Read}_{Y}=(\underline{\mathbf{O 0 . . 1 5}})^{\left(s-21-2 Q^{2}\right) / 8} \\
& \text { Read }_{\text {Slide }}=(\underline{\mathbf{L O} . .7})^{Q^{2} / 2+6} \cdot \underline{\mathbf{L O} . \mathbf{2}} \text {. }
\end{aligned}
$$

Bricks. The role of $\mathbf{R}$ is to read an input $x=x_{q-1} x_{q-2} \cdots x_{1} x_{0}$ from its northwest neighbor and $y=$ $y_{q-1} y_{q-2} \cdots y_{1} y_{0}$ from its southwest neighbor (if the cell is in the vertically-mirrored position, the roles of northeast and southeast positions are swapped) and to create the offset $\Delta_{x y}=2 Q \sum_{i=0}^{q-1}\left(2^{i} x_{i}\right)+2 \sum_{j=0}^{q-1}\left(2^{j} y_{j}\right)$. This module adopts a class of $Q^{2}$ conformations that can be represented as the following parameterized conformation by these inputs.

Let $\Delta_{x}=2 Q \sum_{i=0}^{q-1}\left(2^{i} x_{i}\right)$ and define reusable subpaths:

$$
\begin{aligned}
& \text { PathRFGunit }=\left(\underset{\mathrm{SW}}{\swarrow}, \overrightarrow{\mathrm{E}}, \stackrel{\swarrow \mathrm{SW}}{\overleftarrow{\mathrm{W}})^{4},}\right. \\
& \text { PathRFG }(b, k)= \begin{cases}\text { PathRFGunit } & \text { if } b=0 \\
\left(\left(\mathrm{SW}_{\mathrm{SW}}\right)^{16}\right)^{2^{k-2}} & \text { if } b=1\end{cases}
\end{aligned}
$$

Being parameterized by the inputs $x$ and $y$, the whole path can be represented as:

$$
\operatorname{PathR}(x, y)=(\mathrm{SE}, \overleftarrow{\mathrm{W}})^{4} \cdot \operatorname{PathRFG}^{\mu_{q}} \cdot \operatorname{PathRX}(x) \cdot \operatorname{PathRTurn}(x) \cdot \circlearrowleft_{\pi / 3}(\operatorname{PathRY}(x, y)) \cdot \operatorname{PathRSlide}(x, y)
$$

where

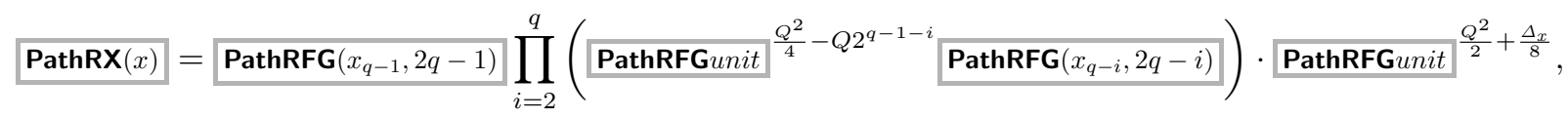

$$
\begin{aligned}
& \operatorname{PathRTurn}(x)=(\mathrm{SE}, \overleftarrow{\mathrm{W}})^{2 Q^{2}-\Delta_{x}+6}(\underset{\mathrm{SW}}{\swarrow}, \overrightarrow{\mathrm{E}})^{7+\Delta_{x}} \\
& \operatorname{PathRY~}(x, y)=\text { PathRFGunit } \overbrace{(s-5) / 8-q Q^{2} / 4-Q / 4-3-\Delta_{x} / 8}^{=\Lambda / 8+\left(8 Q^{2}-\Delta_{x}-2 Q\right) / 8} \\
& \text { PathRFG }\left(y_{q-1}, q-1\right) \prod_{j=2}^{q}\left(\operatorname{PathRYFGunit~}^{\frac{Q^{2}}{4}-2^{q-1-j}}{\operatorname{PathRFG}\left(y_{q-j}, q-j\right)}\right) \cdot \text { PathRFG } u n i t^{1+\frac{\Delta_{x y}}{8}}, \\
& \text { PathRSlide } \left.\left.(x, y)=(\overrightarrow{\mathrm{E}}, \underset{\mathrm{SW}}{\swarrow})^{2 Q^{2}+11-\Delta_{x y}} \underset{\mathrm{E}}{\rightarrow} \underset{\nearrow}{\mathrm{NE}}(\underset{\mathrm{SE}}{\searrow})^{\mathrm{NE}}\right) \underset{\nwarrow}{10}(\underset{\mathrm{E}}{\nwarrow})^{\mathrm{NW}}\right)^{3+\Delta_{x y}} .
\end{aligned}
$$

Along this parameterized path, the transcript $\mathbf{R}$ folds as :

$$
\mathbf{R} !(x, y)=\text { Conformation }(\mathbf{R}, \operatorname{PathR}(x, y)) \text {. }
$$

Fact 3 When a supercell starts at $(0,0)$, reading $x$ and $y$ from the northwest and southwest neighbors lets $\mathbf{R !}(x, y)$ end at $(s+8) \overrightarrow{\mathrm{se}}+\left(s-16-\Delta_{x y}\right) \overrightarrow{\mathrm{sw}}$ and $\mathbf{\mathbf { L }}$ start at its northwestern neighbor $(s+7) \overrightarrow{\mathrm{se}}+\left(s-16-\Delta_{x y}\right) \overrightarrow{\mathrm{sw}}$. 


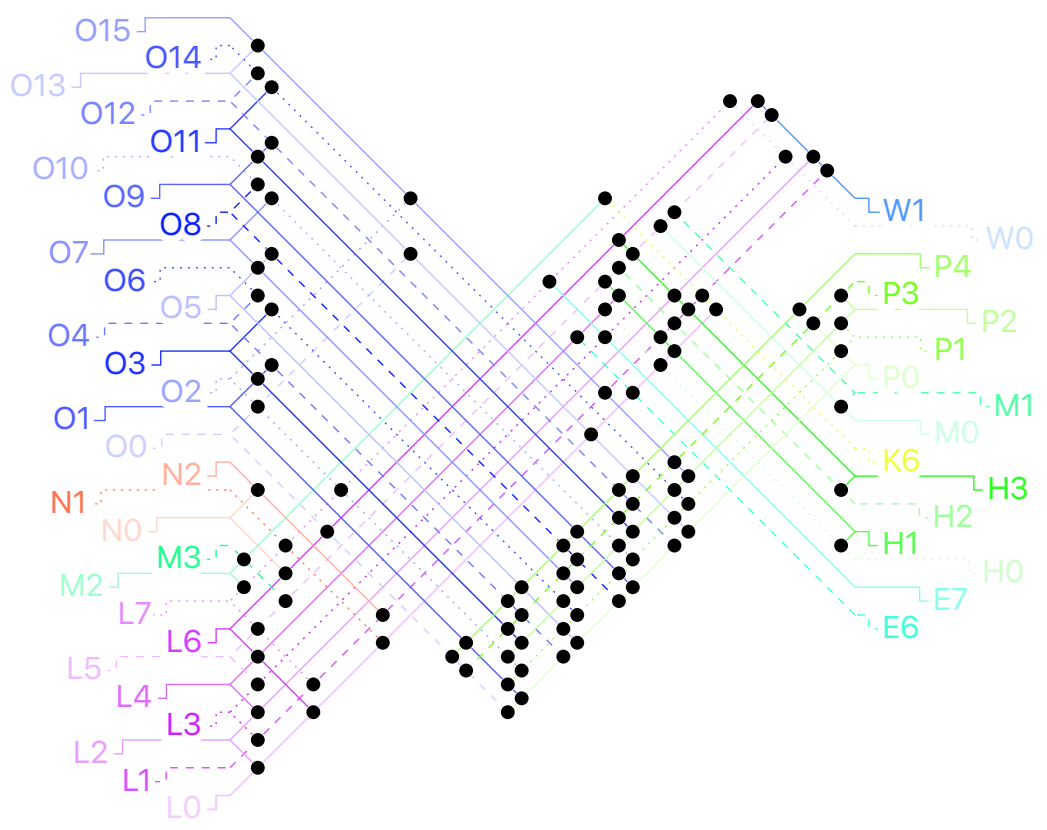

Fig. 17: Subrule for Module R.

Subrule. Figure 17 illustrates the subrule for the interactions between the beads of $\mathbf{R}$ and those of the other modules. 


\section{B.5 Lookup Table}

Transcript length: $2 s-6 Q^{2}-10$

\# of bricks: $Q^{2}$

\# of bead types: 3

Bead types: Q0..2

Transcript. For ease of description, based on the transition function of the simulated S2FA, let us define the following two functions:

$$
\begin{aligned}
& \underline{\mathbf{Q}}_{x^{\prime}}(x, y, i)=\left\{\begin{array}{ll}
\frac{\mathbf{Q} 0^{2}}{\mathbf{Q 1}^{2}} & \text { if the } i \text {-th least-significant bit of } x^{\prime}(x, y) \text { is } 0
\end{array},\right. \\
& \underline{\mathbf{Q}}_{y^{\prime}}(x, y, i)= \begin{cases}\underline{\mathbf{Q}}^{2} & \text { if the } i \text {-th LSB of } y^{\prime}(x, y) \text { is } 0 \\
\underline{\mathbf{Q 1}}^{2} & \text { otherwise }\end{cases}
\end{aligned}
$$

The transcript of this module is

$$
\mathbf{L}=(\underline{\mathbf{Q} 2})_{s-7-\left(2 q Q^{2}+4 \operatorname{InitY}\right)}^{=6+6 Q^{2}+\Lambda} \cdot \text { Lookup } Y^{s-\text { Lookup }_{\text {Turn }}} \cdot \text { Lookup }_{X} \cdot \underline{\mathbf{Q 2}}^{4},
$$

where

$$
\begin{aligned}
& \underline{\text { Lookup }_{Y}}=\prod_{j=0}^{q-1} \prod_{x=0}^{Q-1} \prod_{y=0}^{Q-1} \underline{\mathbf{Q}}_{y^{\prime}}(Q-1-x, Q-1-y, q-j), \\
& \text { Lookup }_{\text {Turn }}=\underline{\mathbf{Q 2}}^{4 \text { InitY }+s-7-6 Q^{2}-2 q Q^{2}}=\underline{\mathbf{Q 2}}^{4 Q^{2}+38+\Lambda} \\
& \text { Lookup }_{X}=\prod_{i=0}^{q-1} \prod_{x=0}^{Q-1} \prod_{y=0}^{Q-1} \mathbf{Q}_{x^{\prime}}(Q-1-x, Q-1-y, q-i) \text {. }
\end{aligned}
$$

Bricks. This module adopts a class of $Q^{2}$ bricks which can be fully described by the following metaconformation parameterized by the offset $\Delta_{x y}=2(Q x+y)$ :

$$
\mathbf{L} !\left(\Delta_{x y}\right)=\text { Conformation }\left(\underline{\mathbf{L}},\left(\mathrm{NE}_{\nearrow}\right)^{s-8-\Delta_{x y}}\left(\nwarrow^{\mathrm{NW}}\right)^{s-3-6 Q^{2}+\Delta_{x y}}\right) .
$$

This is just a straight line which goes northeast first, turns around the east corner of the scaffold prepared by $\mathbf{S}$, and then goes northwest, being pushed upward initially by the previous module $\mathbf{R}$ with the offset $\Delta_{x y}$.

Subrule. Figure 18 illustrates the subrule for the interactions between $\mathbf{Q 0}, \mathbf{Q 1}$, and $\mathbf{Q 2}$, which constitute $\mathbf{L}$, and beads of the other modules. It also includes subrule for SB, which we shall describe next. 


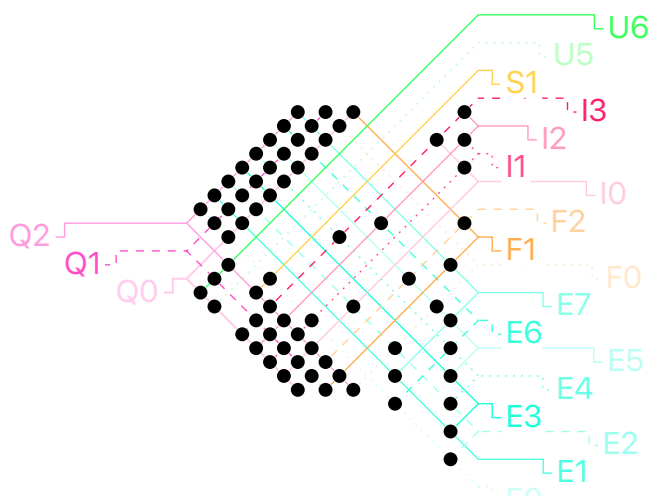

Fig. 18: Subrule for Module L. 


\section{B.6 Speedbump}

Transcript length: $6 Q^{2}+4$

\# of bricks: $Q^{2}$

\# of bead types: 7

Bead types: $\underline{\mathbf{Q} 2}, \mathbf{R 0 . . 1}, \mathbf{S 0 . . 3}$.

Transcript. The transcript of this module is

$$
\mathbf{S B}=\prod_{i=0}^{2 q-1}\left((\mathbf{R 0 . . 1})^{2^{2 q-i}} \cdot \underline{\mathbf{R 0}} \cdot \underline{\mathbf{Q} 2^{2^{2 q-i}-1}}\right)(\mathbf{R 0 . . 1})^{2} \cdot \mathbf{R 0} \cdot \underline{\mathbf{Q 2}} \cdot \underline{\mathbf{S 0}} . . \mathbf{3}
$$

Bricks. This module adopts a class of $Q^{2}$ bricks which can be fully described by the following metaconformation parameterized by $x$ and $y$ :

$$
\text { SB! }(x, y)=\text { Conformation }(\underline{\text { SB },}, \operatorname{PathSB}(x, y)) \text {, }
$$

where using the floor function $h(n)=\lfloor n / 2\rfloor, \operatorname{PathSB}(x, y)\rfloor$ is defined as:

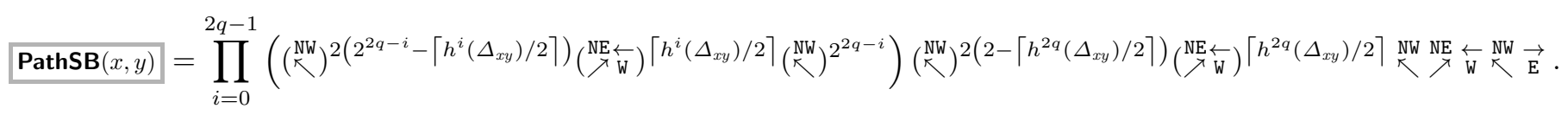

Subrule. Figure 19 illustrates the subrule for the interactions between the beads of Speedbump and those of other modules.

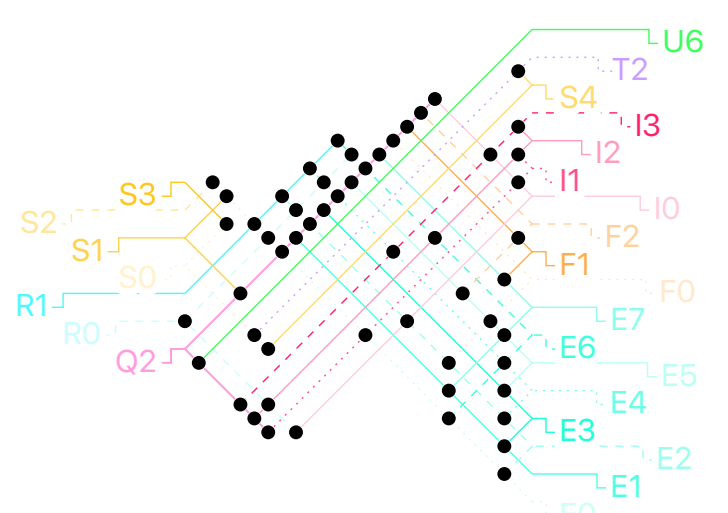

Fig. 19: Subrule for Module SB. 


\section{B.7 Write}

Transcript length: $4 s+10 q+7$

\# of bricks: $Q^{2}$

\# of bead types: 36

Bead types: M0..1, P0..4, S4, T0..7, U0..8, V0..7, W0..2.

Transcript. The transcript of this module is

$$
\mathbf{W}=\operatorname{SegW}_{X} \cdot \operatorname{SegW}_{\text {Sticky }} \cdot \operatorname{SegW}_{Y} \cdot \underline{\mathbf{W} 2} \cdot \mathbf{W O}^{. .1^{6}}
$$

where

$$
\begin{aligned}
& \mathrm{SegFF}_{\text {Init }}=\mathrm{U} 0 . .5 \cdot \mathrm{T} 4 \cdot \mathrm{P} 4 \cdot \mathrm{T6} \cdot \mathrm{P0} \text {, } \\
& \mathrm{SegFF}_{\text {Flap }}=\mathbf{T 0} \cdot \mathbf{P 3} \cdot \mathbf{T 2} \cdot \mathbf{P 2} \cdot \mathbf{T} 4 \cdot \mathbf{P 1} \cdot \mathbf{T 6} \cdot \mathbf{P 0} \text {, } \\
& \text { SegFF }_{T e r m}=\text { U0..4 } \cdot \text { U6..8 } \cdot \text { T5..7, } \\
& \operatorname{SegFF}(\ell)=\operatorname{SegFF}_{\text {Init }} \cdot \operatorname{SegFF}_{\text {Flap }}{ }^{\ell} \cdot \operatorname{SegFF}_{\text {Term }} \text {, } \\
& \mathrm{SegW}_{\text {Xinit }}=\mathrm{S} 4 \cdot \mathrm{M} 0 \cdot \mathrm{T} 2 \cdot \mathrm{M} 1 \cdot \mathrm{T} 4 . .7 \cdot(\mathrm{T0} . .7)^{\mathrm{InitX}} \text {, } \\
& \mathbf{S e g}_{\text {Xterm }}=(\mathbf{T 0 . . 7})^{2 \mu_{q}} \text { T0..1, } \\
& \mathbf{S e g W}_{X}=\operatorname{SegW}_{\text {Xinit }} \cdot\left(\prod_{i=0}^{q-2}\left(\operatorname{SegFF}\left(Q 2^{i}\right) \cdot(\mathbf{T 0 . . 7})^{Q^{2} / 2-Q 2^{i}-2}\right) \cdot \operatorname{SegFF(Q^{2}/2)}\right) \cdot \operatorname{SegW}_{X t e r m}, \\
& \text { SegW }_{\text {Sticky }}=\mathbf{V} \mathbf{0 . . 7 ^ { \text { InitL } / 4 }} \\
& \mathbf{S e g W}_{\text {Yinit }}=\underline{\text { T1..7 }} \cdot(\underline{\text { T0..7 }})^{\text {InitY }-1} \text {, } \\
& \mathbf{S e g W}_{\text {Yterm }}=(\underline{\mathbf{T 0 . . 7}})^{2 Q^{2}-Q / 2+\Lambda / 4} \cdot \underline{\mathbf{T 0 . . 4}} \text {, } \\
& \operatorname{SegW}_{Y}=\operatorname{SegW}_{\text {Yinit }} \cdot\left(\prod_{j=0}^{q-2}\left(\underline{\operatorname{SegFF}\left(2^{j}\right)} \cdot(\underline{\mathbf{T O} 0 . .7})^{Q^{2} / 2-2^{j}-2}\right) \cdot \operatorname{SegFF}(Q / 2)\right) \cdot \operatorname{SegW}_{\text {Yterm }} .
\end{aligned}
$$

Bricks. The module outputs $x^{\prime}(x, y)=x_{q-1}^{\prime} x_{q-2}^{\prime} \cdots x_{1}^{\prime} x_{0}^{\prime}$ and $y^{\prime}(x, y)=y_{q-1}^{\prime} y_{q-2}^{\prime} \cdots y_{1}^{\prime} y_{0}^{\prime}$ by adopting a class of $Q^{2}$ bricks that can be fully described by the following conformation parameterized by $x$ and $y$ :

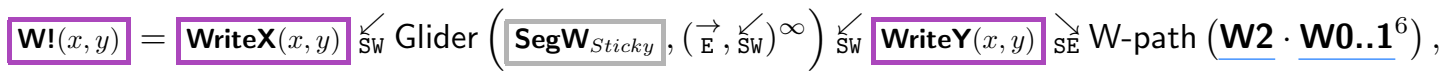

which is equipped with the following two output interfaces:

$$
\begin{aligned}
& \text { WriteX }(x, y)=\operatorname{Glider}\left(\widehat{\operatorname{Seg} \mathbf{W}_{\text {Xinit }}},(\overrightarrow{\mathrm{E}}, \stackrel{\swarrow}{\text { SW }})^{\infty}\right) \text {, }
\end{aligned}
$$

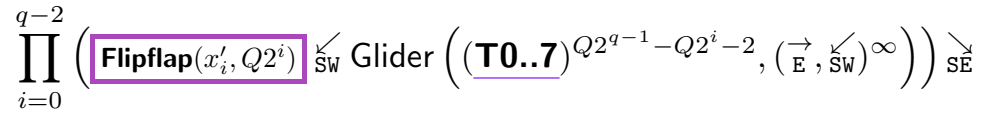

$$
\begin{aligned}
& \text { Flipflap }\left(x_{q-1}^{\prime}, Q 2^{q-1}\right) \text { SW Glider }\left(\operatorname{SegW}_{X t e r m},\left(\overrightarrow{\mathrm{E}}, \breve{\mathrm{SW}}^{\infty}\right),\right.
\end{aligned}
$$

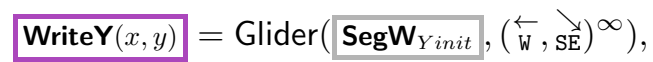

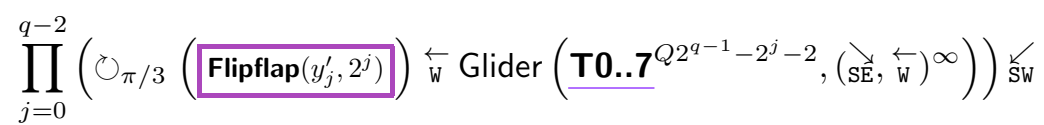

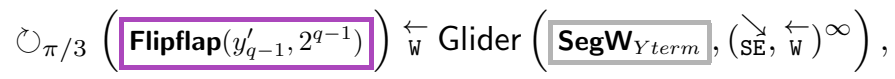


where

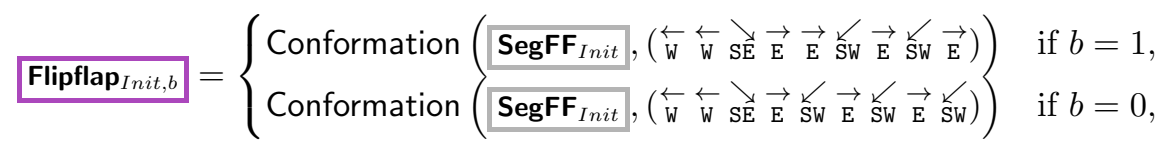

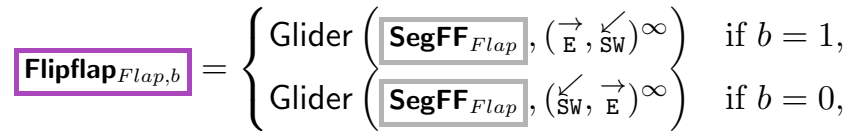

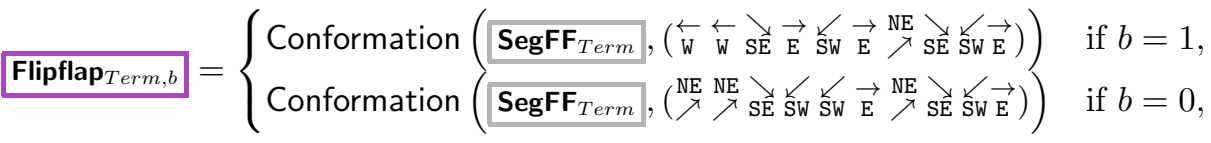

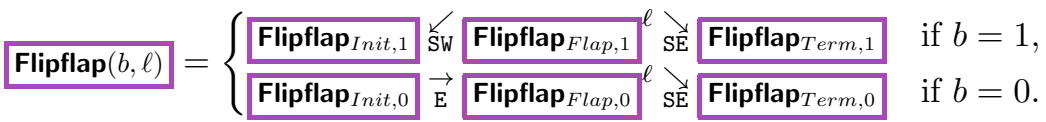

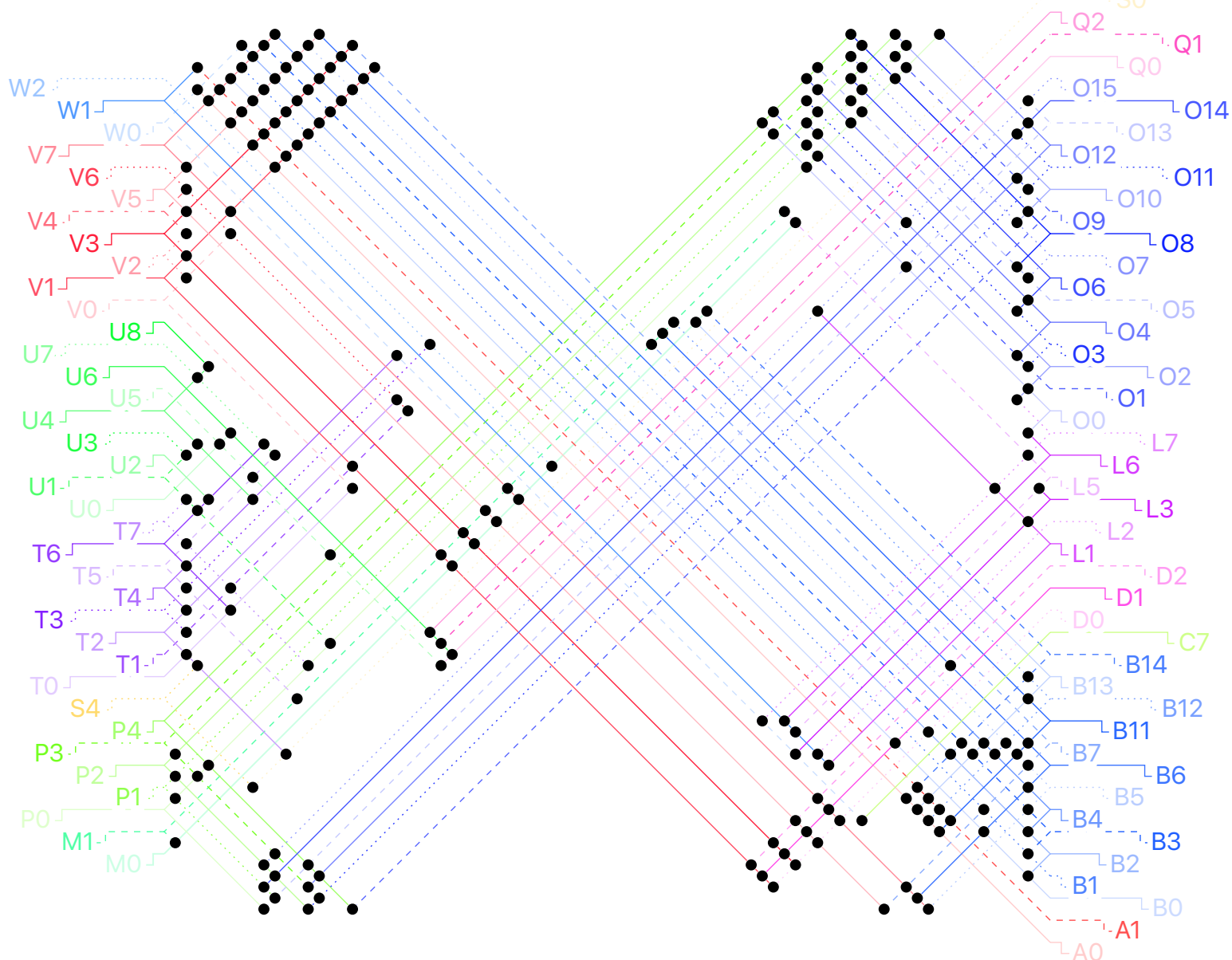

Fig. 20: Subrule for Module $\mathbf{W}$.

Subrule. Figure 20 illustrates the subrule for the interactions between the beads of $\mathbf{W}$ and the beads of the other modules. 


\section{B.8 The seed brick}

The following conformation provides a supercell with northwestern and southwestern neighbors encoding $x$ and $y$ as sequence of $4 Q x$ and $4 y$ magnetic beads each respectively, encoding thus $x$ and $y$ as expected by the read module:

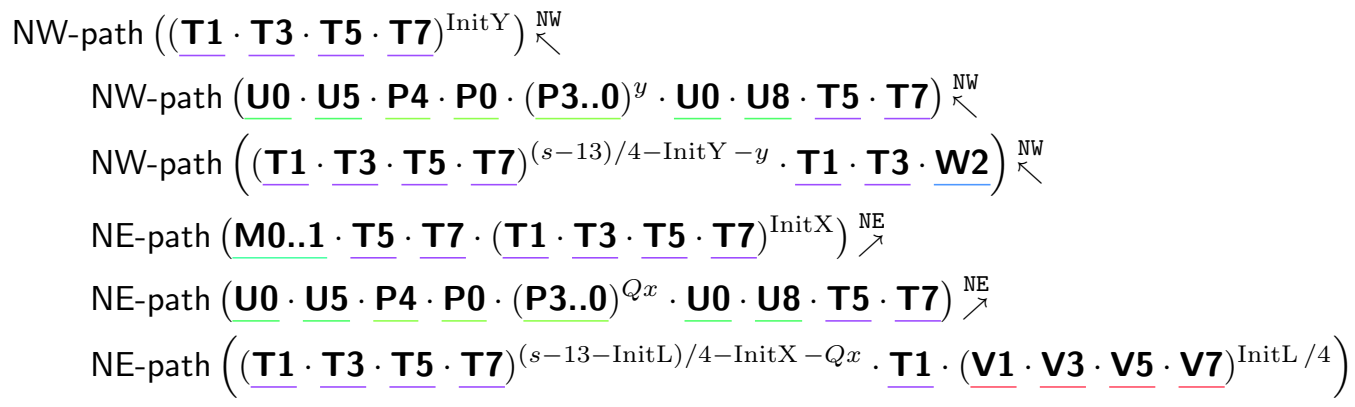

Concatenating as many copies of this conformation as needed by $\nwarrow^{\text {NW }}$ and terminating it with $\stackrel{\text { NW }}{\nwarrow} \mathbf{T} \mathbf{1}{ }_{\nwarrow}^{\text {NW }}$ E-path $\left(\underline{\mathbf{T 3}} \cdot(\underline{\mathbf{L 1}} \cdot \underline{\mathbf{L 3}} \cdot \underline{\mathbf{L 5}} \cdot \underline{\mathbf{L 7}})^{2}\left\langle\underline{\mathbf{L 2} @ 00\rangle)} \underline{\mathrm{SE}} \mathrm{W}\right.\right.$-path $\left(\underline{\mathbf{W 0 . . 1}}{ }^{4}\right)$ yields a seed. 


\section{Module alignment: correctness of the folding}

Figure 21 shows that all modules are correctly aligned and fold thus as expected, whatever the value $x, y \in[Q]$ are, i.e. whatever the offset $\Delta_{x y} \in 2\left[Q^{2}\right]$ is. The following lemmas prove that the figure is indeed correct.

Alignment of supercells We adopt the (east, southwest)-coordinate system in the triangular grid. Let $\overrightarrow{n w}=$ $(-1,-1), \overrightarrow{n e}=(0,-1), \vec{e}=(1,0), \overrightarrow{s e}=(1,1), \overrightarrow{s w}=(0,1)$ and $\vec{w}=(-1,0)$ be the six unit vectors of the triangular grid, so that coordinates $(u, v)=u \overrightarrow{\mathrm{e}}+v \overrightarrow{\mathrm{sw}}$. We will express the positions of the various key beads in each module using these vectors.

Lemma 2. When a supercell starts folding in its upright conformation at $(0,0)$, the next supercell starts at $(s-1)(\overrightarrow{\mathrm{sw}}+\overrightarrow{\mathrm{se}})$ if $\square$ of the current supercell adopts the brick conformation $\mathbf{\square s b}$, and at $12 \overrightarrow{\mathrm{e}}+(s-1) \overrightarrow{\mathrm{ne}}$ otherwise.

Lemma 3. When a supercell starts folding rather in its vertically-mirrored conformation at $(0,0)$, the next supercell starts at $(s-1) \overrightarrow{\mathrm{nw}}+(s-1) \overrightarrow{\mathrm{ne}}$ if $\mathbf{\square}$ of the current supercell adopts the brick conformation $\mathbf{\mathbf { I s b }}$, and at $12 \overrightarrow{\mathrm{e}}+(s-1) \overrightarrow{\mathrm{se}}$ otherwise.

Alignment between flipflaps and flattable gliders Suppose a supercell starts folding in its upright position at $(0,0)$ and now is about to read $x_{q-1} x_{q-2} \cdots x_{1} x_{0}$ from the adjacent $x$-output side of the supercell bit by bit in decreasing order of significance. The $x$-output side is equipped with the $q$ flipflaps, each of which has a unique $\mathbf{U} 8$ bead. Let $P_{q-1}, P_{q-2}, \ldots, P_{1}, P_{0}$ be the points where these beads are; indeed, $P_{q-i}=$ $(-1,0)+\left(\right.$ Init $\left.\vec{L}+8 \mu_{q}+3+\left(2 i-2^{2-i}\right) Q^{2}\right) \overrightarrow{\mathrm{sw}}$.

Lemma 4. For $1 \leqslant i \leqslant q$, let

$$
m_{i}=1+\mu_{q}+\frac{\left(2 i-2^{2-i}\right) Q^{2}}{8}-\frac{Q\left(\sum_{k=q-i+1}^{q-1} 2^{k} x_{k}\right)}{4} .
$$

The bead at the point $P_{q-i}+\overrightarrow{\mathrm{se}}+\overrightarrow{\mathrm{sw}}$ is of type $\underline{\mathbf{0 0}}$ and it is the $m_{i}$-th $\underline{\mathbf{0 0}}$ of $\mathbf{\operatorname { R e a d }}_{X}$.

Proof. Up to $P_{q-1}(i=0), \operatorname{Read}_{X}$ folds uniquely and $m_{1}$-th $\mathbf{O 0}$ is put at $P_{q-1}+\overrightarrow{\mathrm{se}}+\overrightarrow{\mathrm{sw}}$. Suppose $q \geqslant 2$. For $1 \leqslant i<q-1$, the distance between $P_{q-(i+1)}+\overrightarrow{\mathrm{se}}+\overrightarrow{\mathrm{sw}}$ and $P_{q-i}+\overrightarrow{\mathrm{se}}+\overrightarrow{\mathrm{sw}}$ is $2 Q^{2}+2^{q+1} 2^{q-i}$, and hence, a multiple of 8 , and so is the offset created by $x_{q-1} \cdots x_{q-i+1}, 2 Q\left(\sum_{k=q-i+1}^{q-1} 2^{k} x_{k}\right)$ because $Q=2^{q}$ is a multiple of 4 .

The flipflap for the LSB requires $2^{q-1}$ more repetitions of $\mathbf{0 0 . . 1 5}$ after the $m_{q}$-th one and this amount of repetitions is enough. $\operatorname{Read}_{X}$ has $\mu_{q}+(q / 4+1 / 2) Q^{2}$ repetitions of it in total, and hence, is repetitive enough to read all the bits as

$$
\mu_{q}+\frac{q+2}{4} Q^{2}-m_{q} \geqslant \frac{q+2}{4} Q^{2}-1-\frac{2 q-2^{2-q}}{8} Q^{2}=\frac{1}{2} Q^{2}-1 \geqslant 2^{q-1} .
$$

A simple substitution based on these numbers and Lemma 4 suffices to confirm that $\mathbf{R}$ reads the $x$-input correctly.

Now let us consider the reading of $y_{q-1} y_{q-2} \cdots y_{1} y_{0}$ from the adjacent $y$-output side of the supercell bit by bit in decreasing order of significance. The $y$-output side is also equipped with the $q$ flipflaps, each of which has a unique $\mathbf{U 8}$ bead. Let $P_{q-1}^{\prime}, P_{q-2}^{\prime}, \ldots, P_{1}^{\prime}, P_{0}^{\prime}$ be the points where these beads are:

$$
P_{q-i}^{\prime}=(-1, s-1)+\left(6+6 Q^{2}+\frac{2 q Q^{2}+32 \mu_{q}}{3}+2 i Q^{2}-2^{2-i} Q\right) \overrightarrow{\mathrm{se}} .
$$

Lemma 5. For $1 \leqslant i \leqslant q$, let

$$
n_{i}=1+\frac{i+3}{4} Q^{2}-2^{-i-1} Q+\frac{q Q^{2}+16 \mu_{q}}{12}-\frac{\Delta_{x}}{8}-\frac{\sum_{k=q-i+1}^{q-1} 2^{k} y_{k}}{4} \in \mathbb{N} / 2
$$

The bead at the point $P_{q-i}^{\prime}+\overrightarrow{\mathrm{e}}+\overrightarrow{\mathrm{se}}$ is the $n_{i}$-th $\underline{\mathbf{O 0}}$ of $\mathbf{R e a d}_{Y}$ if $n_{i}$ is an integer or the $\left(n_{i}-\frac{1}{2}\right)$-th $\underline{\mathbf{O 8}}$ of $\operatorname{Read}_{Y}$ otherwise. 


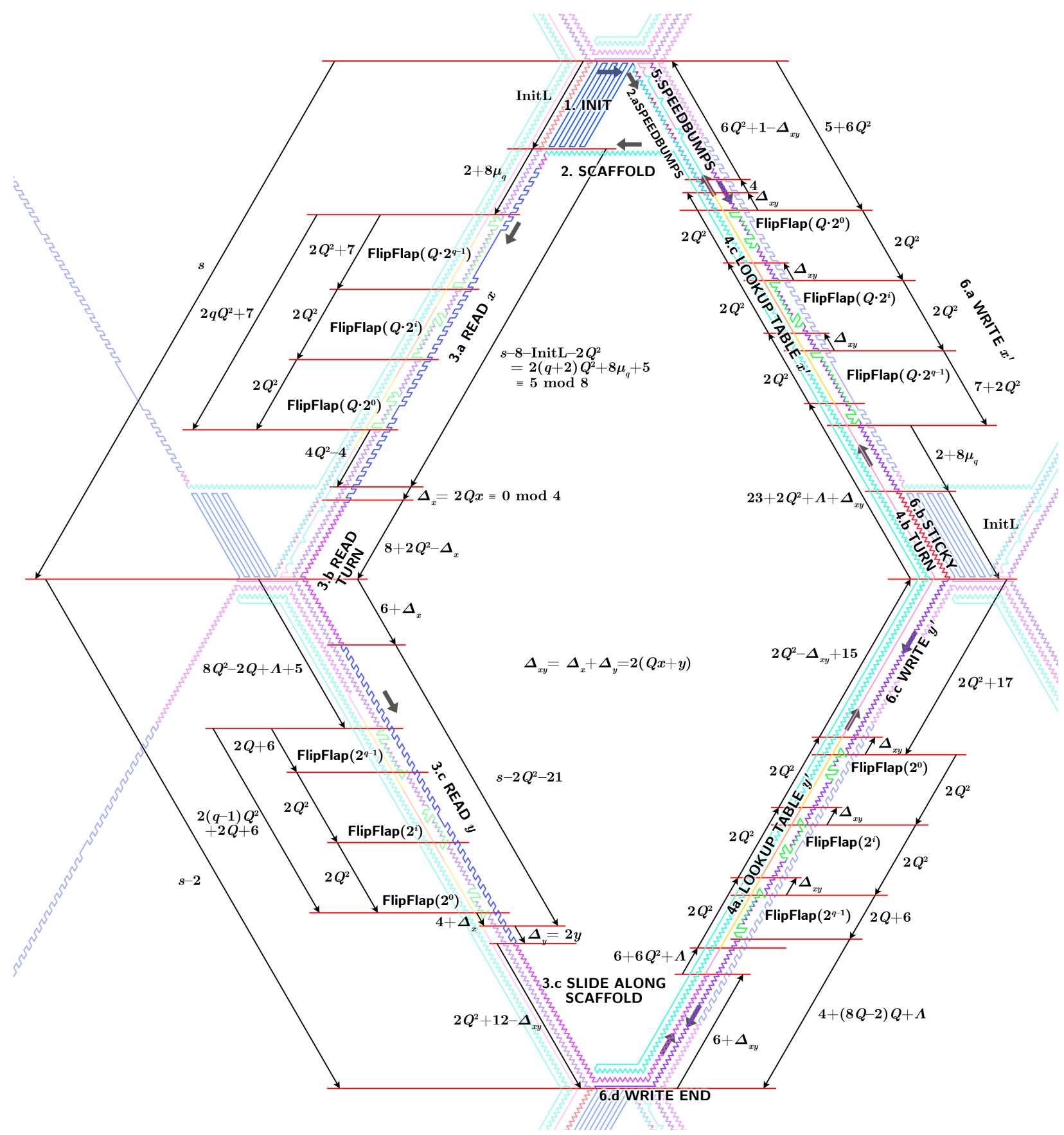

Fig. 21: Alignment of the various modules 
The flipflap for the LSB does not require any more repetition of $\mathbf{0 0 . . 1 5}$ after the $n_{q}$-th one. $\operatorname{Read}_{Y}$ has $1+4 \mu_{q} / 3+(4 q+9) Q^{2} / 12$ repetitions of it in total, and hence, is repetitive enough to read all the bits as

$$
1+\frac{4 \mu_{q}}{3}+\frac{4 q+9}{12} Q^{2}-n_{q} \geqslant \frac{1}{2} \text {. }
$$

Speedbumps The northeastern side of $\mathbf{S}$ is equipped with $2 q+1$ speedbumps, which consist of four bead types 10, I1, 12, and 13. We index them in order of transcription as 0th, 1st, and so on, that is, the last one is $(2 q)$-th. The 0 th speedbump consists of 2 beads, and for $1 \leqslant i \leqslant 2 q$, the $i$-th one consists of $2^{i}$ beads. $\mathbf{S}$ is hardcoded so as to adopt the unique brick, on which the 0th speedbump ranges from $(9,2)$ to $(10,3)$ and for $1 \leqslant i \leqslant 2 q$, the $i$-th one ranges from $(15,8)+6\left(2^{i-1}-1\right) \overrightarrow{\mathrm{se}}$ to $(15,8)+6\left(2^{i-1}-1\right) \overrightarrow{\mathrm{se}}+\left(2^{i}-1\right) \overrightarrow{\mathrm{se}}$.

$\mathbf{L}$ is equipped with $2 q+1$ repetitions of $\mathbf{R} \mathbf{2 . . 3}$ of maximal length, which we call speedbumpees. We also index them in order of transcription as 0th, 1st, and so on. When $\Delta_{x y}=0$, speedbumpees do not overlap with any speedbump at all as illustrated in Figure $5 \mathrm{a}$. Otherwise, for any $0 \leqslant i \leqslant 2 q$, the $(2 q-i)$-th speedbumpee may overlap with the $i$-th speedbump (indeed, never with another speedbump).

For an integer $x$, let us define a function $h$ as $h(x)=\lfloor x / 2\rfloor$. We inductively claim that the $(2 q-i)$-th speedbumpee starts at $(15,7)+6\left(2^{i}-1\right) \overrightarrow{\mathrm{se}}+h^{2 q-i}\left(\Delta_{x y}\right) \overrightarrow{\mathrm{nw}}$. The base case $i=2 q$ can be easily verified from the fact that when $\Delta_{x y}=0$, the 0th speedbumpee starts at $(15,7)+6\left(2^{2 q}-1\right) \overrightarrow{\text { se }}$ and the offset moves this start point northwestward. Suppose this claim holds for $i$. The $(2 q-i)$-th speedbumpee is stretched straight up until when the $\mathbf{R} \mathbf{3}$ can be adjacent to the $i$-th speedbump geometrically. That $\mathbf{R} \mathbf{3}$ and all the following R3's rather repel and form a bump. Note that the speedbumpee begins and ends with $\mathbf{R 2}$, and $\mathbf{R} \mathbf{2}$ and $\mathbf{R} \mathbf{3}$ alternate. Thus, the number of R3's that do not bump is

$$
\left\lfloor\frac{6 \cdot 2^{i}+1-h^{2 q-i}\left(\Delta_{x y}\right)-\left(2^{i+2}+1\right)}{2}\right\rfloor=\left\lfloor\frac{2^{i+1}-h^{2 q-i}\left(\Delta_{x y}\right)}{2}\right\rfloor=2^{i}-\left\lceil\frac{h^{2 q-i}\left(\Delta_{x y}\right)}{2}\right\rceil .
$$

The $(2 q-i)$-th speedbumpee involves $2^{i} \mathbf{R} 3$ 's so that the remaining $\left\lceil h^{2 q-i}\left(\Delta_{x y}\right) / 2\right\rceil \mathbf{R} 3$ 's do bump. Thus, this speedbumpee ends at $(15,7)+6\left(2^{i}-1\right) \overrightarrow{\mathrm{se}}+h^{2 q-i}\left(\Delta_{x y}\right) \overrightarrow{\mathrm{nw}}+2^{i+1} \overrightarrow{\mathrm{nw}}+\left\lceil h^{2 q-i}\left(\Delta_{x y}\right) / 2\right\rceil \overrightarrow{\mathrm{se}}$. Since it is followed by $2^{i}-1 \mathbf{Q} \mathbf{1}$ 's, the next speedbumpee starts at

$$
\begin{aligned}
& (15,7)+\left(6\left(2^{i}-1\right)-2^{i+1}-2^{i}\right) \overrightarrow{\mathrm{se}}+\left(h^{2 q-i}\left(\Delta_{x y}\right)-\left\lceil\frac{h^{2 q-i}\left(\Delta_{x y}\right)}{2}\right\rceil\right) \overrightarrow{\mathrm{nw}} \\
= & (15,7)+6\left(2^{i-1}-1\right) \overrightarrow{\mathrm{se}}+\left\lfloor\frac{h^{2 q-i}\left(\Delta_{x y}\right)}{2}\right\rfloor \overrightarrow{\mathrm{nw}} \\
= & (15,7)+6\left(2^{i-1}-1\right) \overrightarrow{\mathrm{se}}+h^{2 q-(i-1)}\left(\Delta_{x y}\right) \overrightarrow{\mathrm{nw}} .
\end{aligned}
$$

Alignment between lookup tables and flipflaps $\mathbf{W}$ is equipped with $2 q$ flipflaps; the first $q$ of them are to read $x^{\prime}(x, y)=x_{q-1}^{\prime} x_{q-2}^{\prime} \cdots x_{1}^{\prime} x_{0}^{\prime}$ bit by bit in increasing order of significance and the remaining $q$ of them are to read $y^{\prime}(x, y)=y_{q-1}^{\prime} y_{q-2}^{\prime} \cdots y_{1}^{\prime} y_{0}^{\prime}$ bit by bit in increasing order of significance. Note that their transcript $\operatorname{SegFF}(\ell)$ involves exactly two U4's no matter what $\ell$ is.

Lemma 6. For $0 \leqslant i \leqslant q-1$, the leading $\mathbf{U} 4$ of the $(i+1)$-th flipflap, which is to read $x_{i}^{\prime}$, is at $(10,0)+$ $\left(5+6 Q^{2}+2 Q^{2} i\right) \overrightarrow{\text { se. }}$. The first bead of Lookup $X_{X}$ is at $(8,0)+\left(5+6 Q^{2}+2 q Q^{2}-\Delta_{x y}\right) \overrightarrow{\mathrm{se}}$ and the $(i+1)$-th bit of $x^{\prime}(x, y)$ is encoded as Lookup $_{X}\left[2 Q^{2}(q-i-1)+2\left(Q^{2}-1\right)-\Delta_{x y}\right]$ and its successor. Thus, these encoding beads are at $(8,0)+\left(7+6 Q^{2}+2 Q^{2} i\right) \overrightarrow{\mathrm{se}}$ and its northwest neighbor, and put $\mathbf{U} \mathbf{5}$ of the flipflap at $(9,0)+$ $\left(6+6 Q^{2}+2 Q^{2} i\right) \overrightarrow{\mathrm{se}}$ if they are $\underline{\mathbf{Q 0}}$, that is, the $i$-th bit of $x^{\prime}(x, y)$ is 0 , or at $\overline{(11,0)}+\left(5+6 Q^{2}+2 Q^{2} i\right) \overrightarrow{\mathrm{se}}$ otherwise.

Lemma 7. For $0 \leqslant j \leqslant q-1$, the leading $\mathbf{U} 4$ of the $(q+j+1)$-th flipflap, which is to read $y_{j}^{\prime}$, is at $(s+9, s-$ $1)+\left(4\right.$ InitY $\left.+1+2 Q^{2} j\right) \overrightarrow{s w}$. The first bead of Lookup $Y_{Y}$ is at $(s+7, s-1)+\left(4\right.$ InitY $\left.-1+2 q Q^{2}-\Delta_{x y}\right) \overrightarrow{\mathbf{s w}}$ and the $(j+1)$-th bit of $y^{\prime}(x, y)$ is encoded as Lookup $Y_{Y}\left[2 Q^{2}(q-j-1)+2\left(Q^{2}-1\right)-\Delta_{x y}\right]$ and its successor. Thus, these encoding beads are at $(s+7, s-1)+\left(4 \mathrm{InitY}+1+2 Q^{2} j\right) \overrightarrow{\mathrm{sw}}$ and its northeast neighbor, and put U5 of the flipflap at $(s+8, s-1)+\left(4 \operatorname{InitY}+1+2 Q^{2} j\right) \overrightarrow{\mathbf{s w}}$ if they are Q0, that is, the $j$-th bit of $y^{\prime}(x, y)$ is 0 , or at $(s+10, s-1)+\left(4\right.$ InitY $\left.+2+2 Q^{2} j\right) \overrightarrow{s w}$ otherwise. 


\section{The complete attraction rule}

Here us the complete attraction rule in text first, and then illustrated as a matrix in Figure 22.

$\mathrm{A} 0 \mathrm{~B} 10, \mathrm{~B} 11, \mathrm{~B} 12, \mathrm{~B} 13, \mathrm{~B} 14, \mathrm{~B} 15, \mathrm{~B} 16, \mathrm{~B} 17, \mathrm{D} 20, \mathrm{D} 21, \mathrm{~V} 1, \mathrm{~V} 3, \mathrm{~V} 5, \mathrm{~V} 7$, W0

$\mathrm{A} 1 \mathrm{~B} 10, \mathrm{~B} 11, \mathrm{~B} 12, \mathrm{~B} 13, \mathrm{~B} 14, \mathrm{~B} 15, \mathrm{~B} 16, \mathrm{~B} 17, \mathrm{D} 20, \mathrm{~V} 1, \mathrm{~V} 3, \mathrm{~V} 5, \mathrm{~V} 7, \mathrm{~W} 1$

$\mathrm{B} 0 \mathrm{~B} 2, \mathrm{~B} 6, \mathrm{~B} 16, \mathrm{~B} 17, \mathrm{~B} 30, \mathrm{~B} 37, \mathrm{D} 1, \mathrm{D} 68, \mathrm{~V} 1, \mathrm{~V} 3, \mathrm{~V} 5, \mathrm{~V} 7$

$\mathrm{B} 1 \mathrm{~B} 3, \mathrm{~B} 7, \mathrm{~B} 15, \mathrm{~B} 16, \mathrm{~B} 36, \mathrm{~B} 37, \mathrm{D} 8, \mathrm{~V} 1, \mathrm{~V} 3, \mathrm{~V} 5, \mathrm{~V} 7$

$\mathrm{B} 2 \mathrm{~B} 0, \mathrm{~B} 4, \mathrm{~B} 14, \mathrm{~B} 15, \mathrm{~B} 35, \mathrm{~B} 36, \mathrm{D} 0, \mathrm{D} 8, \mathrm{~V} 1, \mathrm{~V} 3, \mathrm{~V} 5, \mathrm{~V} 7$, W0

$\mathrm{B} 3 \mathrm{~B} 1, \mathrm{~B} 5, \mathrm{~B} 13, \mathrm{~B} 14, \mathrm{~B} 34, \mathrm{~B} 35, \mathrm{D} 7, \mathrm{D} 8, \mathrm{~V} 1, \mathrm{~V} 3, \mathrm{~V} 5, \mathrm{~V} 7$

$\mathrm{B} 4 \mathrm{~B} 2, \mathrm{~B} 6, \mathrm{~B} 12, \mathrm{~B} 13, \mathrm{~B} 33, \mathrm{~B} 34, \mathrm{D} 6, \mathrm{D} 45, \mathrm{D} 46, \mathrm{~V} 1, \mathrm{~V} 3, \mathrm{~V} 5, \mathrm{~V} 7$

$\mathrm{B} 5 \mathrm{~B} 3, \mathrm{~B} 7, \mathrm{~B} 11, \mathrm{~B} 12, \mathrm{~B} 32, \mathrm{~B} 33, \mathrm{~V} 1, \mathrm{~V} 3, \mathrm{~V} 5, \mathrm{~V} 7$

$\mathrm{B} 6 \mathrm{~B} 0, \mathrm{~B} 4, \mathrm{~B} 10, \mathrm{~B} 11, \mathrm{~B} 31, \mathrm{~B} 32, \mathrm{D} 2, \mathrm{~V} 1, \mathrm{~V} 3, \mathrm{~V} 5, \mathrm{~V} 7$

$\mathrm{B} 7 \mathrm{~B} 1, \mathrm{~B} 5, \mathrm{~B} 10, \mathrm{~B} 17, \mathrm{~B} 30, \mathrm{~B} 31, \mathrm{D} 68, \mathrm{M} 1, \mathrm{~V} 1, \mathrm{~V} 3, \mathrm{~V} 5, \mathrm{~V} 7$

$B 10$ A0, $A 1, B 6, B 7, B 12, B 16, B 20, B 27$

$\mathrm{B} 11$ A0, $\mathrm{A} 1, \mathrm{~B} 5, \mathrm{~B} 6, \mathrm{~B} 13, \mathrm{~B} 17, \mathrm{~B} 26, \mathrm{~B} 27, \mathrm{D} 21, \mathrm{D} 68, \mathrm{D} 69, \mathrm{M} 1$

$\mathrm{B} 12$ A0, $\mathrm{A} 1, \mathrm{~B} 4, \mathrm{~B} 5, \mathrm{~B} 10, \mathrm{~B} 14, \mathrm{~B} 25, \mathrm{~B} 26, \mathrm{D} 27, \mathrm{D} 68, \mathrm{M} 1$

$\mathrm{B} 13 \mathrm{A0}, \mathrm{A} 1, \mathrm{~B} 3, \mathrm{~B} 4, \mathrm{~B} 11, \mathrm{~B} 15, \mathrm{~B} 24, \mathrm{~B} 25, \mathrm{D} 20, \mathrm{D} 26, \mathrm{D} 66, \mathrm{D} 67, \mathrm{M} 0$

$\mathrm{B} 14 \mathrm{A0}, \mathrm{A} 1, \mathrm{~B} 2, \mathrm{~B} 3, \mathrm{~B} 12, \mathrm{~B} 16, \mathrm{~B} 23, \mathrm{~B} 24, \mathrm{D} 27, \mathrm{D} 66, \mathrm{MO}$

$\mathrm{B} 15 \mathrm{~A} 0, \mathrm{~A} 1, \mathrm{~B} 1, \mathrm{~B} 2, \mathrm{~B} 13, \mathrm{~B} 17, \mathrm{~B} 22, \mathrm{~B} 23, \mathrm{D} 25, \mathrm{D} 64, \mathrm{D} 65$

$\mathrm{B} 16$ A0, $\mathrm{A} 1, \mathrm{~B} 0, \mathrm{~B} 1, \mathrm{~B} 10, \mathrm{~B} 14, \mathrm{~B} 21, \mathrm{~B} 22, \mathrm{D} 7$

$B 17 \mathrm{A0}, \mathrm{A} 1, \mathrm{~B} 0, \mathrm{~B} 7, \mathrm{~B} 11, \mathrm{~B} 15, \mathrm{~B} 20, \mathrm{~B} 21, \mathrm{D} 7$

$\mathrm{B} 20 \mathrm{~B} 10, \mathrm{~B} 17, \mathrm{~B} 22, \mathrm{~B} 26, \mathrm{~B} 36, \mathrm{~B} 37, \mathrm{C} 6, \mathrm{C} 7$

$\mathrm{B} 21$ B16,B17,B23,B27,B35,B36, C5, C6, D41

$\mathrm{B} 22 \mathrm{~B} 15, \mathrm{~B} 16, \mathrm{~B} 20, \mathrm{~B} 24, \mathrm{~B} 34, \mathrm{~B} 35, \mathrm{C} 4, \mathrm{C} 5, \mathrm{D} 46$

$\mathrm{B} 23 \mathrm{~B} 14, \mathrm{~B} 15, \mathrm{~B} 21, \mathrm{~B} 25, \mathrm{~B} 33, \mathrm{~B} 34, \mathrm{C} 3, \mathrm{C} 4, \mathrm{D} 7, \mathrm{D} 8, \mathrm{D} 40, \mathrm{D} 46$

$\mathrm{B} 24 \mathrm{~B} 13, \mathrm{~B} 14, \mathrm{~B} 22, \mathrm{~B} 26, \mathrm{~B} 32, \mathrm{~B} 33, \mathrm{C} 2, \mathrm{C} 3, \mathrm{D} 6, \mathrm{D} 45, \mathrm{D} 46$

$\mathrm{B} 25 \mathrm{~B} 12, \mathrm{~B} 13, \mathrm{~B} 23, \mathrm{~B} 27, \mathrm{~B} 31, \mathrm{~B} 32, \mathrm{C} 1, \mathrm{C} 2, \mathrm{D} 5, \mathrm{D} 6, \mathrm{D} 44$

$\mathrm{B} 26 \mathrm{~B} 11, \mathrm{~B} 12, \mathrm{~B} 20, \mathrm{~B} 24, \mathrm{~B} 30, \mathrm{~B} 31, \mathrm{C} 0, \mathrm{C} 1, \mathrm{D} 26$

$\mathrm{B} 27 \mathrm{~B} 10, \mathrm{~B} 11, \mathrm{~B} 21, \mathrm{~B} 25, \mathrm{~B} 30, \mathrm{~B} 37, \mathrm{C} 0, \mathrm{C} 7, \mathrm{D} 26$

$\mathrm{B} 30 \mathrm{~B} 0, \mathrm{~B} 7, \mathrm{~B} 26, \mathrm{~B} 27, \mathrm{~B} 32, \mathrm{~B} 36, \mathrm{D} 61$

$\mathrm{B} 31 \mathrm{~B} 6, \mathrm{~B} 7, \mathrm{~B} 25, \mathrm{~B} 26, \mathrm{~B} 33, \mathrm{~B} 37, \mathrm{D} 69$

B32 B5, B6, B24, B25, B30, B34, D26, D27, D60, D68

$\mathrm{B} 33$ B4,B5,B23,B24,B31,B35,D26, D69

$\mathrm{B} 34 \mathrm{~B} 3, \mathrm{~B} 4, \mathrm{~B} 22, \mathrm{~B} 23, \mathrm{~B} 32, \mathrm{~B} 36, \mathrm{D} 24, \mathrm{D} 25, \mathrm{D} 67$

$\mathrm{B} 35$ B2, B3, B21, B22, B33, B37, C0, D45

$\mathrm{B} 36 \mathrm{~B} 1, \mathrm{~B} 2, \mathrm{~B} 20, \mathrm{~B} 21, \mathrm{~B} 30, \mathrm{~B} 34, \mathrm{D} 45, \mathrm{D} 62$

$\mathrm{B} 37 \mathrm{~B} 0, \mathrm{~B} 1, \mathrm{~B} 20, \mathrm{~B} 27, \mathrm{~B} 31, \mathrm{~B} 35, \mathrm{C} 1$

$\mathrm{C} 0$ B26, $\mathrm{B} 27, \mathrm{~B} 35, \mathrm{C} 2, \mathrm{C} 6$

$\mathrm{C} 1 \mathrm{~B} 25, \mathrm{~B} 26, \mathrm{~B} 37, \mathrm{C} 3, \mathrm{C} 7, \mathrm{D} 27$

$\mathrm{C} 2$ B24,B25,C0,C4,D26,D27

$\mathrm{C} 3 \mathrm{~B} 23, \mathrm{~B} 24, \mathrm{C} 1, \mathrm{C} 5, \mathrm{D} 25, \mathrm{D} 26$

$\mathrm{C} 4 \mathrm{~B} 22, \mathrm{~B} 23, \mathrm{C} 2, \mathrm{C} 6, \mathrm{D} 24, \mathrm{D} 25$

$\mathrm{C} 5 \mathrm{~B} 21, \mathrm{~B} 22, \mathrm{C} 3, \mathrm{C} 7, \mathrm{D} 23, \mathrm{D} 24$

$\mathrm{C} 6 \mathrm{~B} 20, \mathrm{~B} 21, \mathrm{C} 0, \mathrm{C} 4, \mathrm{D} 22, \mathrm{D} 23, \mathrm{E} 0, \mathrm{E} 1$

C7 B20, B27, C1, C5, D21, D22, W0

D0 B2,D5,D44,V1,V3,V5, V7

$\mathrm{D} 1 \mathrm{~B} 0, \mathrm{D} 3, \mathrm{D} 4, \mathrm{D} 43, \mathrm{~V} 1, \mathrm{~V} 3, \mathrm{~V} 5, \mathrm{~V} 7$

$\mathrm{D} 2 \mathrm{~B} 6, \mathrm{D} 42, \mathrm{~V} 1, \mathrm{~V} 3, \mathrm{~V} 5, \mathrm{~V} 7$

D3 D1,D41

$\mathrm{D} 4 \div \mathrm{D} 1, \mathrm{D} 40$

D5 B25,D0

D6 B4,B24,B25

$\mathrm{D} 7 \mathrm{~B} 3, \mathrm{~B} 16, \mathrm{~B} 17, \mathrm{~B} 23$

$\mathrm{D} 8 \mathrm{~B} 1, \mathrm{~B} 2, \mathrm{~B} 3, \mathrm{~B} 23$ 


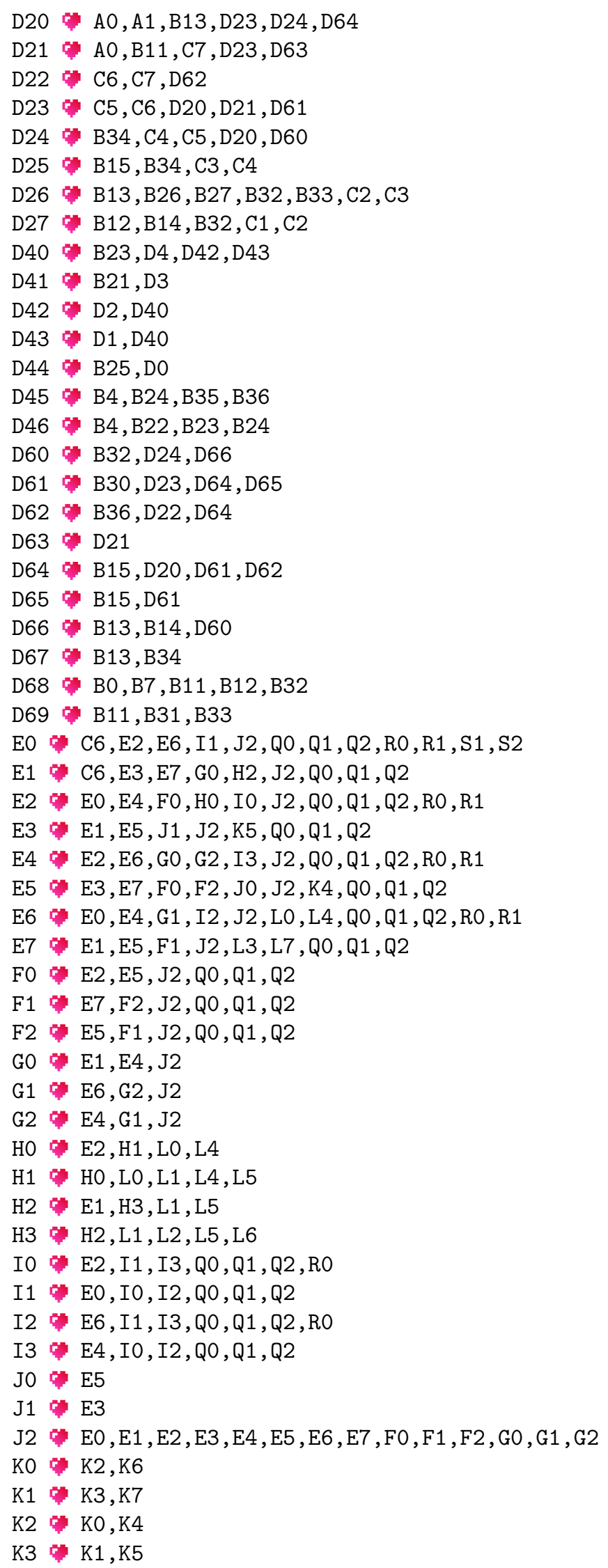




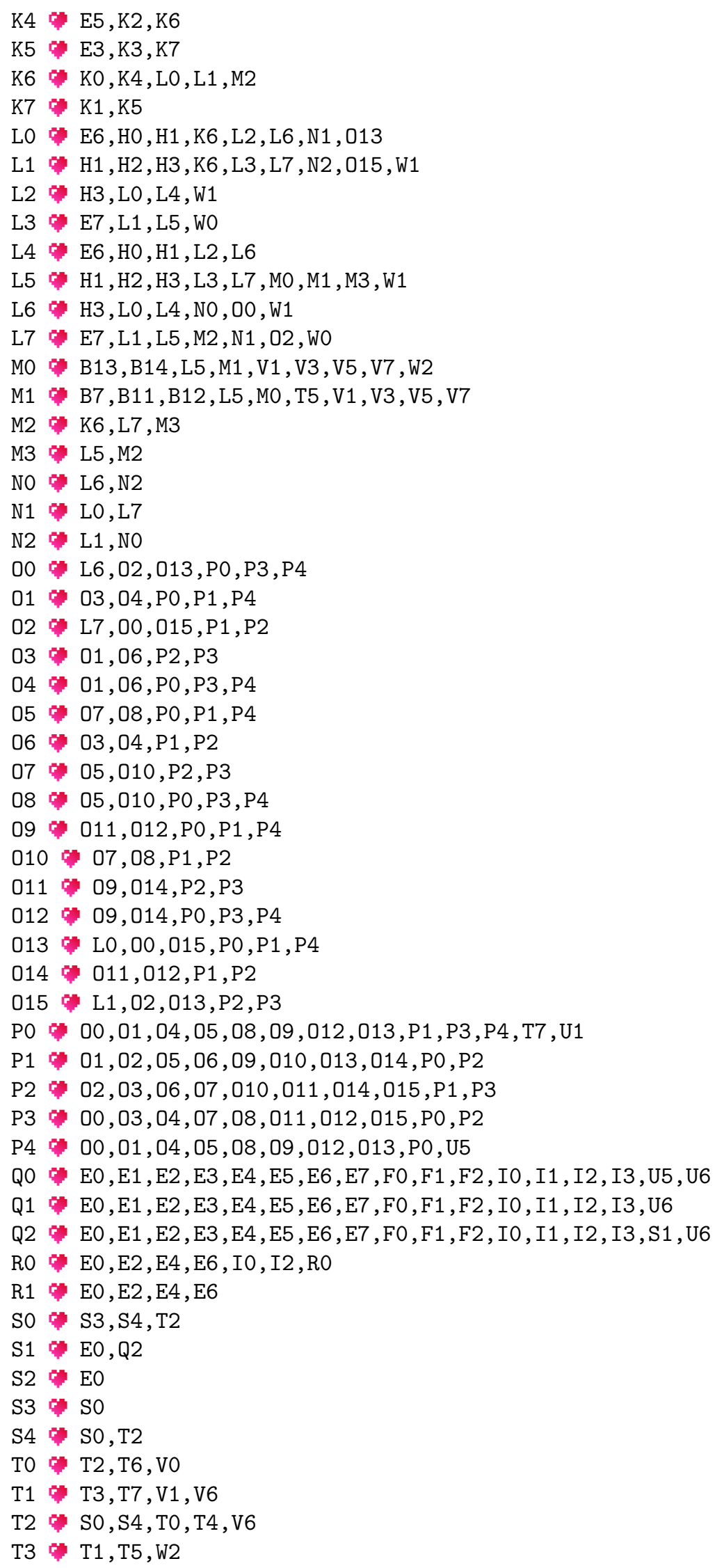




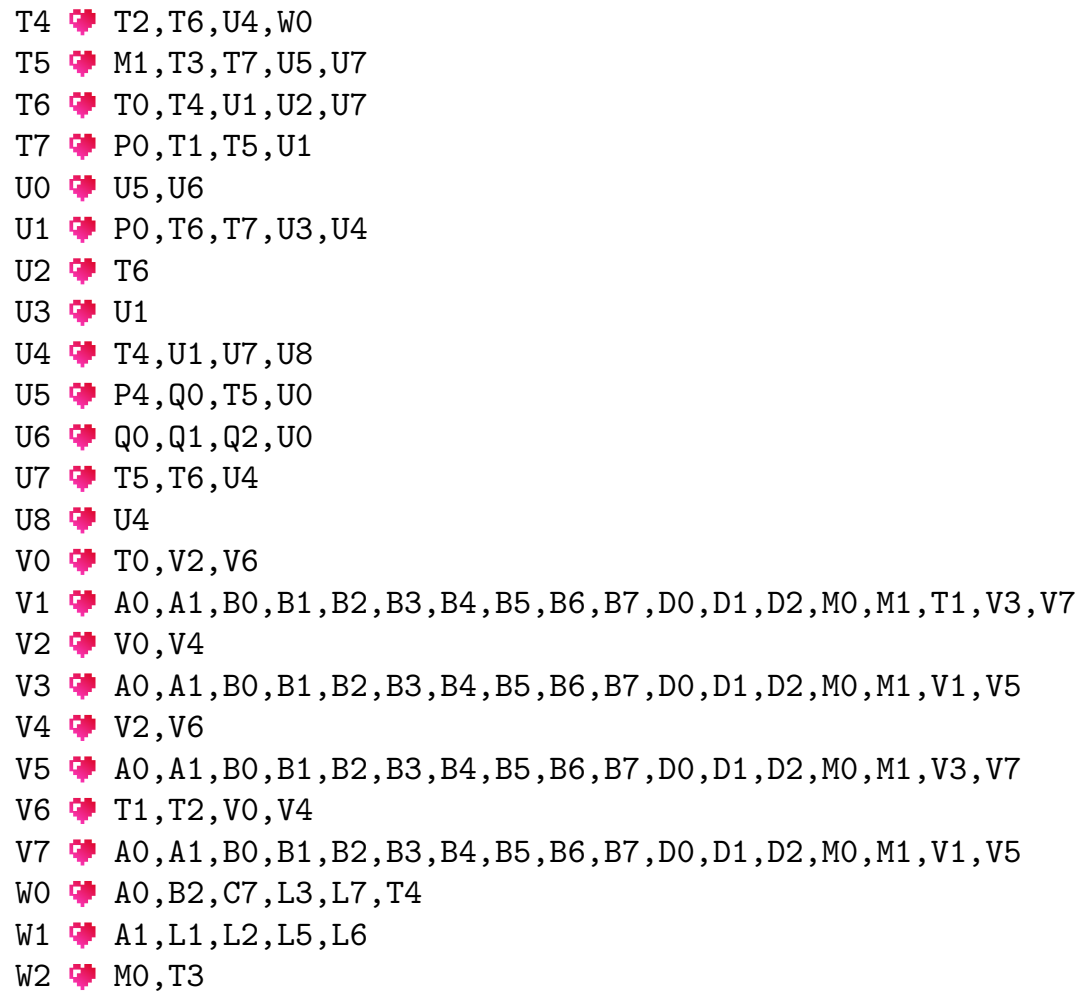




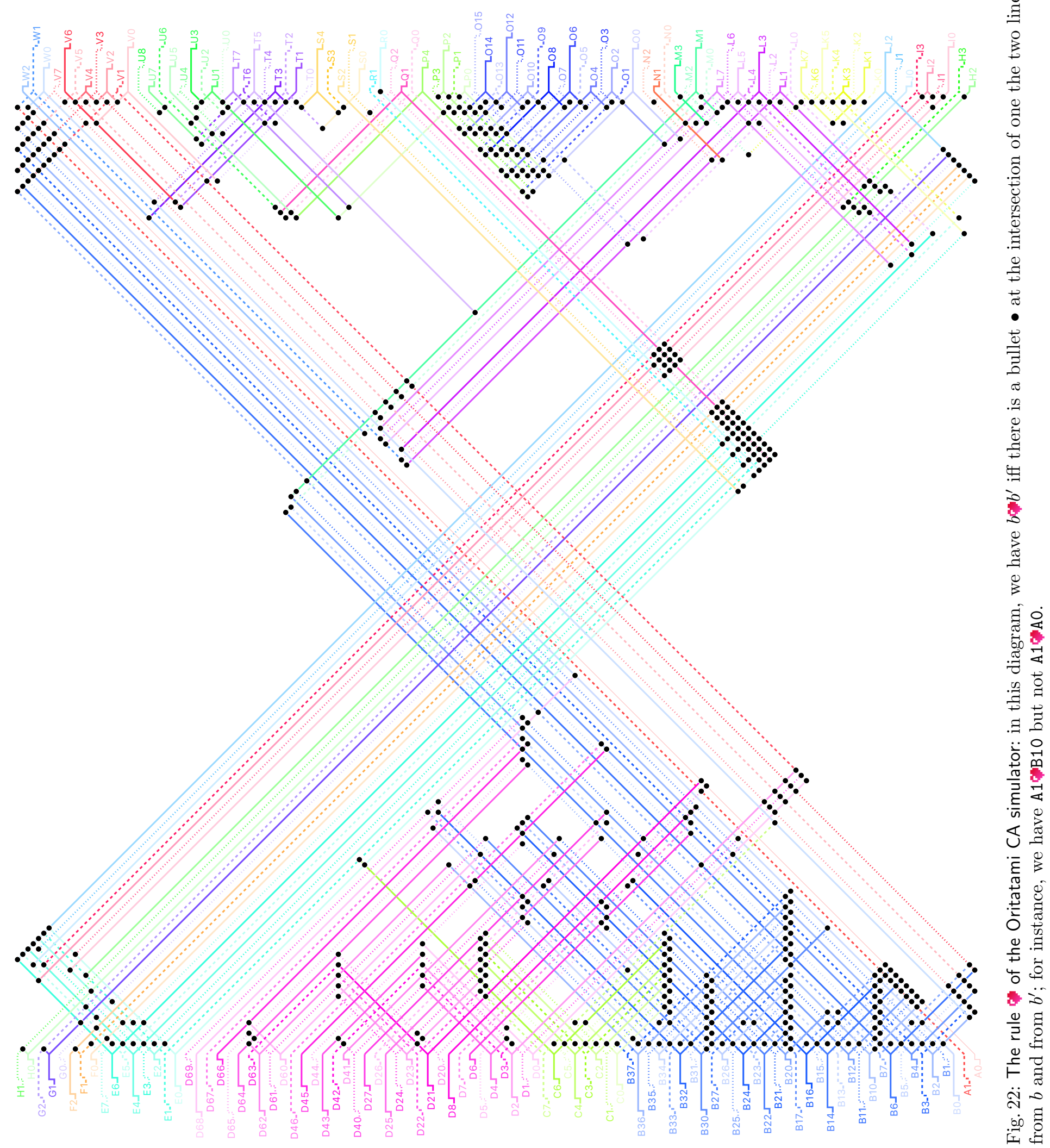

